\title{
CONVERGENCE OF DISCONTINUOUS GALERKIN METHODS FOR INCOMPRESSIBLE TWO-PHASE FLOW IN HETEROGENEOUS MEDIA*
}

\author{
JISHENG $\mathrm{KOU}^{\dagger}$ AND SHUYU SUN $\ddagger$
}

\begin{abstract}
A class of discontinuous Galerkin methods with interior penalties is presented for incompressible two-phase flow in heterogeneous porous media with capillary pressures. The semidiscrete approximate schemes for fully coupled system of two-phase flow are formulated. In highly heterogeneous permeable media, the saturation is discontinuous due to different capillary pressures, and therefore, the proposed methods incorporate the capillary pressures in the pressure equation instead of saturation equation. By introducing a coupling approach for stability and error estimates instead of the conventional separate analysis for pressure and saturation, the stability of the schemes in space and time and a priori $h p$ error estimates are presented in the $L^{2}\left(H^{1}\right)$ for pressure and in the $L^{\infty}\left(L^{2}\right)$ and $L^{2}\left(H^{1}\right)$ for saturation. Two time discretization schemes are introduced for effectively computing the discrete solutions.
\end{abstract}

Key words. two-phase flow, discontinuous Galerkin method, stability, error estimates

AMS subject classifications. 65M60, 65M 15

DOI. $10.1137 / 120898358$

1. Introduction. The discontinuous Galerkin (DG) methods [2, 14, 28, 36, 41] have recently gained great popularity due to their local mass conservative virtue. The DG methods are conveniently applied on unstructured meshes and mesh adaptation, and they utilize the interior penalties to impose the interelement continuity weakly. For more attractive features of DG, we refer to [36]. The DG methods have been widely applied to solve the convection-diffusion equation and transport problems, for example, $[5,15,34,35,36,37]$. The DG methods have been employed to solve the miscible displacement problem in porous media, for example, [12, 30, 38, 42].

Numerical simulation for incompressible two-phase flow in porous media is an important topic in hydrology and petroleum reservoir engineering. Mathematical models of two-phase flow in porous media can be established from the phase mass conservation and Darcy's law. This model consists of a coupled system of nonlinear partial differential equations, in which the nonlinearity arises mainly from relative permeabilities and capillary pressure describing the interactions between the permeable media and the fluids. It has been shown that heterogeneity in capillary pressure has a significant influence on flow paths $[20,21,27,39]$. In heterogeneous media with different distributions of permeability, different capillary pressure functions are employed within the rocks of different permeability type, and as a result, the capillary pressure functions of different permeable media may show the discontinuity on the interface

*Received by the editors November 9, 2012; accepted for publication (in revised form) August 19, 2013; published electronically December 11, 2013. The authors were supported by the National Natural Science Foundation of China (11301163) and the Key Project of Chinese Ministry of Education (212109).

http://www.siam.org/journals/sinum/51-6/89835.html

${ }^{\dagger}$ School of Mathematics and Statistics, Hubei Engineering University, Xiaogan 432000, Hubei, China (jkou@hbeu.edu.cn).

${ }^{\ddagger}$ Corresponding author. Computational Transport Phenomena Laboratory, Division of Physical Science and Engineering, King Abdullah University of Science and Technology, Thuwal 23955-6900, Kingdom of Saudi Arabia (shuyu.sun@kaust.edu.sa).

3280 
of rocks. In this case, however, the capillary pressure is continuous, and therefore, the continuity of capillary pressure results in discontinuity of saturation [21]. The classical fractional flow formulation (for example, used in $[7,8,9,17]$ ) incorporates the capillarity into the saturation equation and requires the continuity of saturation in spatial dimension. This formulation cannot be well used for the case of different capillary pressure functions for multiple rock types, because of the discontinuity of saturation across the rock interface [21].

Recently, a two-phase flow formulation has been introduced in [21], which is based on the conception that the wetting-phase pressure is always continuous as long as none of the phases are immobile, and can correctly describe discontinuity in the saturation due to continuity of the capillary pressure and discontinuity of different capillary pressure functions on the interface of regions. The main difference from the classical model is that this formulation incorporates the capillary pressure in the pressure equation instead of the saturation equation. Based on the fact that the saturation is discontinuous in the entire domain, the DG methods are preferable because they use discontinuous polynomial spaces. In this paper, based on the formulation used in [21], we will propose DG schemes for both the pressure and saturation equations.

A number of numerical methods for simulating two-phase flow in porous media have been developed in the literature, for example, [1, 13, 21, 22, 32, 33, 39]. The coupled mixed finite element and Galerkin methods or finite volume methods have been presented for two-phase flow in [8, 29]. In [19, 21, 24, 26], the combined mixed finite element and DG methods have recently been employed to compute the two-phase flow problems. Based on the traditional formulation of two-phase flow, a fully coupled DG scheme for both the pressure and saturation equations is proposed in [16, 18], and the convergence and error estimates of numerical solutions are analyzed in [17]. Differently from the work in $[16,17]$, we will propose and analyze the fully coupled DG schemes for both the pressure and saturation equations based on the two-phase flow formulation used in [21], which is often used in practical applications, but with great difficulty for mathematical analysis of numerical methods.

According to the two-phase flow formulation used in [21], the capillarity is incorporated into the pressure equation instead of the saturation equation. In the classical two-phase flow formulation, the capillarity is reformed into the saturation equation as a diffusion term of saturation, which can facilitate the theoretical analysis of the numerical schemes. Unlike the cases of the classical formulation, the analysis of the methods based on the up to date two-phase flow formulation is much more elusive. The main technical difficulty is the lack of control on the saturation in the pressure equation, which arises from capillarity. Another difficulty is the analysis of the nonlinear intrinsic relationship between pressure and saturation. The technique used in [17] treats the pressure and saturation equations separately, but it has great difficulty in dealing with the broken gradient of saturation in the pressure equation. We will introduce a coupling approach by virtue of the coupling relationship between the pressure equation and the saturation equation, and analyze the error estimates of the proposed schemes. The stability of numerical solutions and a priori $h p$ error estimates are obtained in the $L^{2}\left(H^{1}\right)$ for the pressure and in the $L^{\infty}\left(L^{2}\right)$ and $L^{2}\left(H^{1}\right)$ for the saturation.

The rest of this paper is organized as follows. In section 2, we describe the twophase flow model and the formulation proposed in [21]. In section 3, the fully coupled DG methods for two-phase flow are presented. The stability of numerical schemes is analyzed in section 4. A priori error analysis of numerical schemes is carried out in section 5. The global existence of discrete solutions is demonstrated in section 6 , 
and two time discretization schemes are introduced to effectively compute the discrete solutions in section 7 . Concluding remarks are provided in section 8 .

2. Mathematical model. The mathematical model of incompressible and immiscible two-phase fluid flow in porous media is established in the phase mass conservation law, Darcy's law, and the constraint relations [9]. The wetting phase is denoted by a subscript $w$, and the nonwetting phase is denoted by $n$. We denote by $\phi$ the porosity of the medium, the saturation of phase $\alpha$ by $S_{\alpha}$, and Darcy's velocity of phase $\alpha$ by $\mathbf{u}_{\alpha}$. Let $q_{\alpha}$ be the external mass flow rate of phase $\alpha$, and the mass conservation equation of each phase is given by

$$
\phi \frac{\partial S_{\alpha}}{\partial t}+\nabla \cdot \mathbf{u}_{\alpha}=q_{\alpha}, \quad \alpha=w, n .
$$

Darcy's velocity of each phase is described as

$$
\mathbf{u}_{\alpha}=-\frac{k_{r \alpha}}{\mu_{\alpha}} \mathbf{K}\left(\nabla p_{\alpha}+\rho_{\alpha} g \nabla z\right), \quad \alpha=w, n,
$$

where $g$ is the gravity acceleration and $z$ is the depth. Here, $\mathbf{K}$ denotes the absolute permeability tensor in a porous medium, and the other coefficients $k_{r \alpha}, \mu_{\alpha}, p_{\alpha}$, and $\rho_{\alpha}$ are the relative permeability, viscosity, pressure, and density of each phase, respectively.

The saturations of two phases satisfy $S_{w}+S_{n}=1$. The capillary pressure is defined as the difference between the wetting phase and nonwetting phase pressures:

$$
p_{c}:=p_{n}-p_{w} .
$$

In practical applications, the capillary pressures are often viewed as the functions of the wetting-phase saturation $S_{w}$. In this paper, we always assume that $p_{c}(S)$ is a decreasing function of $S$ and uniformly bounded.

In this model, the absolute permeability tensor $\mathbf{K}$ and viscosity are given by the properties of a porous medium and fluids, respectively, while the capillary pressure and relative permeabilities are the given functions of the wetting-phase saturation. The primary unknowns are the pressures and saturations of two phases, but the number of unknowns can be reduced to two by using the saturation constraint and capillarity relation. Here, we choose the pressure and saturation of the wetting phase as the principal unknown variables and drop their subscripts for simplicity; that is, let $p:=p_{w}$ and $S:=S_{w}$. For the sake of simplicity, we disregard the effect of gravity, but the results presented in this paper can be suitable to the case involving gravity. We define the phase mobility as $\lambda_{\alpha}:=k_{r \alpha} / \mu_{\alpha}$ and the total mobility as $\lambda_{t}:=\lambda_{w}+\lambda_{n}$.

Summing the mass conservation equations of two phases, and using Darcy's velocities of two phases and the capillary pressure definition, we can obtain

$$
-\nabla \cdot\left(\lambda_{t} \mathbf{K} \nabla p\right)-\nabla \cdot\left(\lambda_{n} \mathbf{K} \nabla p_{c}\right)=q_{t},
$$

where $q_{t}:=q_{w}+q_{n}$. The combination of mass conservation equation and Darcy's velocity of the wetting phase gives us

$$
\phi \frac{\partial S}{\partial t}-\nabla \cdot\left(\lambda_{w} \mathbf{K} \nabla p\right)=q_{w} .
$$

The coupled system consisting of (2.4) and (2.5) is the formulation used in [21]. In the above formulation for two-phase flow, we can use (2.4) to compute the pressure 
of the wetting phase, so it is called the pressure equation; we use (2.5) to update the saturation and call it the saturation equation.

Assume that $\Omega \subset \mathbb{R}^{d}(d=2,3)$ is a convex, polygonal, and bounded domain. The boundary $\partial \Omega$ of the domain is divided into two parts $\Gamma_{D}$ and $\Gamma_{N}$, and $\partial \Omega=\bar{\Gamma}_{D} \bigcup \bar{\Gamma}_{N}$. The boundary conditions for the pressure and the saturation are given by

$$
\begin{gathered}
S=S_{B}, \quad p=p_{B} \quad \text { on } \Gamma_{D}, \\
-\lambda_{w} \mathbf{K} \nabla p \cdot \mathbf{n}=-\lambda_{n} \mathbf{K} \nabla p_{n} \cdot \mathbf{n}=0 \quad \text { on } \Gamma_{N} .
\end{gathered}
$$

It is clear that $\Gamma_{D}$ denotes both inflow and outflow boundaries, whereas $\Gamma_{N}$ indicates the no-flow boundary. It follows by the relation between the wetting-phase and no-wetting-phase pressures that the boundary condition (2.7) implies $-\left(\lambda_{t} \mathbf{K} \nabla p+\right.$ $\left.\lambda_{n} \mathbf{K} \nabla p_{c}\right) \cdot \mathbf{n}=0$ on $\Gamma_{N}$. The initial condition for the saturation is given by

$$
S=S^{0} \text { on } \Omega, \quad t=0 .
$$

We make some assumptions on the above two-phase flow model as below.

(H1) The permeability tensor $\boldsymbol{K}$ is symmetric positive definite. It is uniformly bounded above and below; that is, there exist two positive constants $k^{b}, k^{a}$ such that $k^{b} \xi^{T} \xi \leqslant \xi^{T} \mathbf{K} \xi \leqslant k^{a} \xi^{T} \xi \forall \xi \in \mathbb{R}^{d}$.

(H2) The porosity $\phi$ is time independent and uniformly bounded below and above: $0<\phi^{b} \leqslant \phi \leqslant \phi^{a}$.

(H3) The mobilities are nonnegative and uniformly bounded functions of saturations: $0<\lambda_{t}^{b} \leqslant \lambda_{t}(S) \leqslant \lambda_{t}^{a}, \quad 0 \leqslant \lambda_{w}(S) \leqslant \lambda_{w}^{a}, \quad 0 \leqslant \lambda_{n}(S) \leqslant \lambda_{n}^{a}$.

(H4) $\lambda_{w}(S), \lambda_{n}(S)$, and $\lambda_{t}(S)$ are Lipschitz continuous with respect to $S$.

\section{Discontinuous Galerkin methods for two-phase flow.}

3.1. Notation. Here, the traditional notation is used for the Lebesgue spaces $L^{p}(R)$, the Sobolev spaces $W^{k, p}(R)$ with $1 \leqslant p \leqslant \infty$ over a domain $R$. In particular, $W^{k, 2}(R)$ is denoted by $H^{k}(R)$. The corresponding norms are denoted by $\|\cdot\|_{L^{p}(R)}$, $\|\cdot\|_{W^{k, p}(R)}$, and $\|\cdot\|_{H^{k}(R)}$, respectively. For simplicity, we also denote $\|\cdot\|_{W^{k, p}(R)}$ by $\|\cdot\|_{k, p, R}$ and $\|\cdot\|_{H^{k}(R)}$ by $\|\cdot\|_{k, R}$. The inner product of $L^{p}(\Omega)$ or $\left(L^{p}(\Omega)\right)^{d}$ is denoted by $(\cdot, \cdot)$, and the inner product of the functions in the boundary $\Gamma$ is denoted by $(\cdot, \cdot)_{\Gamma}$. For a given normed space $X$ and $1 \leqslant p \leqslant \infty$, we define

$$
L^{p}(0, T ; X):=\left\{\varphi: \varphi(t) \in X,\|\varphi\|_{X} \in L^{p}(0, T)\right\},
$$

which is a normed linear space equipped with the norm given by

$$
\|\varphi\|_{L^{p}(0, T ; X)}:=\left\|\left(\|\varphi\|_{X}\right)\right\|_{L^{p}(0, T)} .
$$

We use a partition $\mathcal{E}_{h}$ of the domain $\Omega$, which is composed of triangles or quadrilaterals if $d=2$, or tetrahedra, prisms, or hexahedra if $d=3$. Denote by $h_{E}$ the diameter of $E \in \mathcal{E}_{h}$ and let $h=\max _{E \in \mathcal{E}_{h}} h_{E}, h / h_{E} \leqslant C$. We assume that $\mathcal{E}_{h}$ is nondegenerate; that is, each element is convex, and that there exists $\rho>0$ such that each of the subtriangles (for $d=2$ ) or subtetrahedra (for $d=3$ ) of element $E$ contains a ball of radius $\rho h_{E}$ in its interior. We also make an assumption that no element crosses the boundaries of $\Gamma_{D}$ or $\Gamma_{N}$.

For the sake of regularizing the heterogeneity of a porous medium, we assume that there exists a nondegenerate partition $\mathcal{E}_{h}^{0}$ of $\Omega$ such that the absolute permeability tensor $\mathbf{K}$ is a piecewise-constant function within each $E \in \mathcal{E}_{h}^{0}$. In this paper, we use $\mathcal{E}_{h}=\mathcal{E}_{h}^{0}$ or a local refined mesh of $\mathcal{E}_{h}^{0}$. 
The set of all interior edges or faces for $\mathcal{E}_{h}$ is denoted by $\Gamma_{h}$, and the sets of all edges or faces on the boundaries $\Gamma_{D}$ and on $\Gamma_{N}$ for $\mathcal{E}_{h}$ are indicated by $\Gamma_{h, D}$ and $\Gamma_{h, N}$, respectively. For any $\gamma \in \Gamma_{h}$, a unit normal vector $\mathbf{n}_{\gamma}$ is chosen, and for any $\gamma \in \Gamma_{h, D} \bigcup \Gamma_{h, N}$, the normal vector $\mathbf{n}_{\gamma}$ coincides with the outward unit normal vector.

For $s \geqslant 0$, the broken Sobolov space is defined by

$$
H^{s}\left(\mathcal{E}_{h}\right):=\left\{\varphi \in L^{2}(\Omega):\left.\varphi\right|_{E} \in H^{s}(E), E \in \mathcal{E}_{h}\right\} .
$$

In this paper, we often write $H^{s}\left(\mathcal{E}_{h}\right)$ as $H^{s}$ for simplicity. For $0 \leqslant m \leqslant s$, the broken norm is defined as

$$
\|\varphi\|_{m}:=\left(\sum_{E \in \mathcal{E}_{h}}\|\varphi\|_{H^{m}(E)}^{2}\right)^{1 / 2} .
$$

Let $\gamma \in \Gamma_{h}$ be shared by the elements $E_{\gamma}^{1}$ and $E_{\gamma}^{2}$ with $\mathbf{n}_{\gamma}$ exterior to $E_{\gamma}^{1}$; that is, $\gamma=E_{\gamma}^{1} \cap E_{\gamma}^{2}$. The average and jump values for $\varphi \in H^{s}\left(\mathcal{E}_{h}\right)(s>1 / 2)$ on $\gamma$ are defined by

$$
\langle\varphi\rangle:=\frac{1}{2}\left(\left.\left(\left.\varphi\right|_{E_{\gamma}^{1}}\right)\right|_{\gamma}+\left.\left(\left.\varphi\right|_{E_{\gamma}^{2}}\right)\right|_{\gamma}\right), \quad[\varphi]:=\left.\left(\left.\varphi\right|_{E_{\gamma}^{1}}\right)\right|_{\gamma}-\left.\left(\left.\varphi\right|_{E_{\gamma}^{2}}\right)\right|_{\gamma} .
$$

For $\gamma \in \Gamma_{h, D}$, we define $\langle\varphi\rangle:=\left.\varphi\right|_{\gamma}$ and $[\varphi]:=\left.\varphi\right|_{\gamma}$.

The DG methods utilize discontinuous piecewise polynomials to approximate the solutions of differential equations. Let $\mathbb{P}_{r}(E)$ denote the space of polynomials of (total) degree less than or equal to $r$ on the element $E$, then we take the discontinuous finite element space as

$$
\mathcal{D}_{r}\left(\mathcal{E}_{h}\right):=\left\{\varphi \in L^{2}(\Omega):\left.\varphi\right|_{E} \in \mathbb{P}_{r}(E), \quad E \in \mathcal{E}_{h}\right\} .
$$

We now recall the following well-known inverse estimates [10, 31, 40].

Lemma 3.1. Let $E \in \mathcal{E}_{h}$, and $v \in \mathbb{P}_{r}(E)$, then there exists a constant $C$ independent of $v, r$, and $h_{E}$ such that

$$
\left\|D^{q+1} v\right\|_{0, E} \leqslant C \frac{r^{2}}{h_{E}}\left\|D^{q} v\right\|_{0, E}, \quad\left\|D^{q} v\right\|_{0, \partial E} \leqslant C \frac{r}{h_{E}^{1 / 2}}\left\|D^{q} v\right\|_{0, E}, \quad q \geqslant 0 .
$$

Lemma 3.2 (see [6]). For any $v \in \mathcal{D}_{r}\left(\mathcal{E}_{h}\right)$, there exists a constant $C$ independent of $h$ and $r$ such that

$$
\|v\|_{0, \Omega}^{2} \leqslant C\left(\sum_{E \in \mathcal{E}_{h}}\|\nabla v\|_{0, E}^{2}+\sum_{\gamma \in \Gamma_{h}}|\gamma|^{d /(1-d)}\left(\int_{\gamma}[v]\right)^{2}+\left(\int_{\Gamma_{h, D}} v\right)^{2}\right),
$$

where $|\gamma|$ denotes the measure of $\gamma$.

3.2. Continuous-in-time schemes. Here, we aim to find the solutions of pressure and saturation in the broken Sobolov space $H^{1}\left(\mathcal{E}_{h}\right)$ instead of the usual space $H^{1}(\Omega)$. We consider the pressure equation defined within each element

$$
-\nabla \cdot \lambda_{t}(S) \mathbf{K} \nabla p-\nabla \cdot \lambda_{n}(S) \mathbf{K} \nabla p_{c}=q_{t} \quad \text { in } E \in \mathcal{E}_{h} .
$$

Multiplying the equation (3.3) by $\varphi \in H^{1}\left(\mathcal{E}_{h}\right)$ and integrating over each element 
$E \in \mathcal{E}_{h}$, we then apply Green's formula to obtain

$$
\begin{aligned}
\int_{E} \lambda_{t}(S) \mathbf{K} \nabla p \cdot \nabla \varphi-\int_{\partial E} \lambda_{t}(S) \mathbf{K} \nabla p \cdot & \mathbf{n}_{\partial E} \varphi+\int_{E} \lambda_{n}(S) \mathbf{K} \nabla p_{c} \cdot \nabla \varphi \\
& -\int_{\partial E} \lambda_{n}(S) \mathbf{K} \nabla p_{c} \cdot \mathbf{n}_{\partial E} \varphi=\int_{E} q_{t} \varphi .
\end{aligned}
$$

Define two velocity variables as

$$
\mathbf{u}_{a}=-\lambda_{t} \mathbf{K} \nabla p, \quad \mathbf{u}_{c}=-\lambda_{n} \mathbf{K} \nabla p_{c} .
$$

The normal components of these velocities are continuous across the element interfaces [21]. Thus, using the continuity of $\mathbf{u}_{a}$ and $\mathbf{u}_{c}$ and taking into account the boundary conditions, we sum (3.4) over all elements to obtain

$$
\begin{aligned}
& \sum_{E \in \mathcal{E}_{h}} \int_{E} \lambda_{t}(S) \mathbf{K} \nabla p \cdot \nabla \varphi-\sum_{\gamma \in \Gamma_{h}} \int_{\gamma}\left\langle\lambda_{t}(S) \mathbf{K} \nabla p \cdot \mathbf{n}_{\gamma}\right\rangle[\varphi] \\
& -\sum_{\gamma \in \Gamma_{h, D}} \int_{\gamma}\left(\lambda_{t}\left(S_{B}\right) \mathbf{K} \nabla p \cdot \mathbf{n}_{\gamma}\right) \varphi=\int_{\Omega} q_{t} \varphi-\sum_{E \in \mathcal{E}_{h}} \int_{E} \lambda_{n}(S) \mathbf{K} \nabla p_{c} \cdot \nabla \varphi \\
& \quad+\sum_{\gamma \in \Gamma_{h}} \int_{\gamma}\left\langle\lambda_{n}(S) \mathbf{K} \nabla p_{c} \cdot \mathbf{n}_{\gamma}\right\rangle[\varphi]+\sum_{\gamma \in \Gamma_{h, D}} \int_{\gamma}\left(\lambda_{n}\left(S_{B}\right) \mathbf{K} \nabla p_{c} \cdot \mathbf{n}_{\gamma}\right) \varphi .
\end{aligned}
$$

Based on (3.5), we introduce a functional for the pressure equation as

$$
\begin{aligned}
B_{p}(p, \varphi ; S):= & \sum_{E \in \mathcal{E}_{h}} \int_{E} \lambda_{t}(S) \mathbf{K} \nabla p \cdot \nabla \varphi-\sum_{\gamma \in \Gamma_{h}} \int_{\gamma}\left\langle\lambda_{t}(S) \mathbf{K} \nabla p \cdot \mathbf{n}_{\gamma}\right\rangle[\varphi] \\
& -\sum_{\gamma \in \Gamma_{h, D}} \int_{\gamma}\left(\lambda_{t}\left(S_{B}\right) \mathbf{K} \nabla p \cdot \mathbf{n}_{\gamma}\right) \varphi \\
& +s_{\text {form }} \sum_{\gamma \in \Gamma_{h}} \int_{\gamma}\left\langle\lambda_{t}(S) \mathbf{K} \nabla \varphi \cdot \mathbf{n}_{\gamma}\right\rangle[p] \\
& +s_{\text {form }} \sum_{\gamma \in \Gamma_{h}, D} \int_{\gamma}\left(\lambda_{t}\left(S_{B}\right) \mathbf{K} \nabla \varphi \cdot \mathbf{n}_{\gamma}\right) p+J_{0, D}^{\sigma}(p, \varphi) .
\end{aligned}
$$

In our schemes, the wetting-phase saturation is permitted to be discontinuous on the element interfaces, but it is continuous within each element and its broken gradient over each element is well defined. Since the absolute permeability is element wise constant and the capillary pressure is a function of saturation, we have $\nabla p_{c}=$ $p_{c}^{\prime}(S) \nabla S$ within each element, which is apparently valid for (3.5). We also define a functional for the pressure equation

$$
\begin{aligned}
L_{p}(\varphi ; S):= & \int_{\Omega} q_{t} \varphi-\sum_{E \in \mathcal{E}_{h}} \int_{E} \lambda_{c}(S) \mathbf{K} \nabla S \cdot \nabla \varphi+\sum_{\gamma \in \Gamma_{h}} \int_{\gamma}\left\langle\lambda_{c}(S) \mathbf{K} \nabla S \cdot \mathbf{n}_{\gamma}\right\rangle[\varphi] \\
& +\sum_{\gamma \in \Gamma_{h, D}} \int_{\gamma}\left(\lambda_{c}\left(S_{B}\right) \mathbf{K} \nabla S \cdot \mathbf{n}_{\gamma}\right) \varphi \\
& -s_{\text {form }} \sum_{\gamma \in \Gamma_{h}} \int_{\gamma}\left\langle\lambda_{n}(S) \mathbf{K} \nabla \varphi \cdot \mathbf{n}_{\gamma}\right\rangle\left[p_{c}(S)\right] \\
& -J_{0}^{\sigma}\left(p_{c}(S), \varphi\right)+s_{\text {form }} \sum_{\gamma \in \Gamma_{h, D}} \int_{\gamma} \lambda_{t}\left(S_{B}\right) \mathbf{K} \nabla \varphi \cdot \mathbf{n}_{\gamma} p_{B}+J_{D}^{\sigma}\left(p_{B}, \varphi\right),
\end{aligned}
$$

Copyright $@$ by SIAM. Unauthorized reproduction of this article is prohibited. 
where

$$
\lambda_{c}(S):=\lambda_{n}(S) p_{c}^{\prime}(S)
$$

Let $\gamma \in \Gamma_{h}$ be shared by the two elements $E_{\gamma}^{1}$ and $E_{\gamma}^{2}$ with $\mathbf{n}_{\gamma}$ exterior to $E_{\gamma}^{1}$; then the upwind value of saturation $\left.S^{*}\right|_{\gamma}$ can be defined as

$$
\left.S^{*}\right|_{\gamma}=\left\{\begin{array}{lll}
\left.S\right|_{E_{\gamma}^{1}} & \text { if } & \mathbf{u}_{a} \cdot \mathbf{n}_{\gamma} \geqslant 0 \\
\left.S\right|_{E_{\gamma}^{2}} & \text { if } & \mathbf{u}_{a} \cdot \mathbf{n}_{\gamma}<0
\end{array}\right.
$$

If $\gamma \in \Gamma_{h, D}$, then we take $\left.S^{*}\right|_{\gamma}=S_{B}$. We further define two functions of the saturation $S$ as

$$
f_{w}(S):=\lambda_{w}(S) / \lambda_{t}(S), \quad f_{n}(S):=\lambda_{n}(S) / \lambda_{t}(S) .
$$

Similarly to [18], we introduce a nonlinear functional argument with the upwind scheme for the discrete saturation equation, which is defined by

$$
\begin{aligned}
(3.10) B_{S}(S, \varphi ; p):= & \sum_{E \in \mathcal{E}_{h}} \int_{E} \lambda_{w}(S) \mathbf{K} \nabla p \cdot \nabla \varphi-\sum_{\gamma \in \Gamma_{h}} \int_{\gamma} f_{w}\left(S^{*}\right)\left\langle\lambda_{t}(S) \mathbf{K} \nabla p \cdot \mathbf{n}_{\gamma}\right\rangle[\varphi] \\
& -\sum_{\gamma \in \Gamma_{h, D}} \int_{\gamma} \lambda_{w}\left(S_{B}\right) \mathbf{K} \nabla p \cdot \mathbf{n}_{\gamma} \varphi-\omega J_{0}^{\sigma}\left(p_{c}(S), \varphi\right) \\
& +s_{\text {form }} \sum_{\gamma \in \Gamma_{h}} \int_{\gamma} f_{w}\left(S^{*}\right)\left\langle\lambda_{t}(S) \mathbf{K} \nabla \varphi \cdot \mathbf{n}_{\gamma}\right\rangle[p] \\
& +s_{\text {form }} \sum_{\gamma \in \Gamma_{h, D}} \int_{\gamma} \lambda_{w}\left(S_{B}\right) \mathbf{K} \nabla \varphi \cdot \mathbf{n}_{\gamma} p+J_{0, D}^{\sigma}(p, \varphi)+J_{D}^{\sigma}(S, \varphi) .
\end{aligned}
$$

We also define a functional for the saturation equation as

$$
\begin{aligned}
L_{S}(\varphi):=s_{\text {form }} \sum_{\gamma \in \Gamma_{h, D}} \int_{\gamma} \lambda_{w}\left(S_{B}\right) \mathbf{K} \nabla \varphi & \cdot \mathbf{n}_{\gamma} p_{B} \\
& +J_{D}^{\sigma}\left(p_{B}, \varphi\right)+J_{D}^{\sigma}\left(S_{B}, \varphi\right)+\int_{\Omega} q_{w} \varphi .
\end{aligned}
$$

In (3.6), (3.7), (3.10), and (3.11), we take $s_{\text {form }} \in\{-1,0,1\}$. It is noted that $s_{\text {form }}=-1$ for the Oden-Babuška-Baumann DG (OBB-DG) formulation [28] and nonsymmetric interior penalty Galerkin [31], $s_{\text {form }}=0$ for incomplete interior penalty Galerkin [14], and $s_{\text {form }}=1$ for symmetric interior penalty Galerkin [41]. In (3.10), $\omega$ is a positive constant and one choice is given in section 5 .

The interior penalty terms in our schemes are defined by

$$
\begin{aligned}
J_{0}^{\sigma}(\psi, \varphi) & :=\sum_{\gamma \in \Gamma_{h}} \frac{r^{2} \sigma_{\gamma}}{h_{\gamma}} \int_{\gamma}[\psi][\varphi], \quad J_{D}^{\sigma}(\psi, \varphi):=\sum_{\gamma \in \Gamma_{h, D}} \frac{r^{2} \sigma_{\gamma}}{h_{\gamma}} \int_{\gamma} \psi \varphi, \\
J_{0, D}^{\sigma}(\psi, \varphi) & :=J_{0}^{\sigma}(\psi, \varphi)+J_{D}^{\sigma}(\psi, \varphi),
\end{aligned}
$$

where $\sigma$ is a discrete positive function that takes the constant value $\sigma_{\gamma}$ on the edge $\gamma$. Although there is no penalty term in OBB-DG $(\sigma=0), \sigma$ is always assumed to be bounded below and above, i.e., $0<\sigma_{0} \leqslant \sigma \leqslant \sigma_{m}$, for the analysis of DG schemes in 
this paper. We note that the saturation may be discontinuous at the element interfaces of $\mathcal{E}_{h}^{0}$, but the pressures are always continuous throughout the entire region, and as a result, the penalty terms are imposed on the wetting-phase pressure $p$ and the capillary pressure $p_{c}$, but the saturation is free of the penalty except at the boundary of the domain.

Based on the bilinear forms and linear functionals defined above and letting $J=$ $(0, T]$ denote the time interval, we now state the schemes of DG for the pressure equation and the saturation equation: find continuous-in-time DG approximations $p_{h}(\cdot, t) \in D_{r}\left(\mathcal{E}_{h}\right)$ and $S_{h}(\cdot, t) \in \mathcal{D}_{r}\left(\mathcal{E}_{h}\right)$ such that

$$
\begin{aligned}
B_{p}\left(p_{h}, \varphi ; S_{h}\right) & =L_{p}\left(\varphi ; S_{h}\right), & & \varphi \in \mathcal{D}_{r}\left(\mathcal{E}_{h}\right), \quad t \in J, \\
\left(\phi \frac{\partial S_{h}}{\partial t}, \varphi\right)+B_{S}\left(S_{h}, \varphi ; p_{h}\right) & =L_{S}(\varphi), & & \varphi \in \mathcal{D}_{r}\left(\mathcal{E}_{h}\right), \quad t \in J, \\
\left(S_{h}, \varphi\right) & =\left(S^{0}, \varphi\right), & & \varphi \in \mathcal{D}_{r}\left(\mathcal{E}_{h}\right), \quad t=0 .
\end{aligned}
$$

In (3.13), the upwind values of $\left.S_{h}^{*}\right|_{\gamma}$ are determined by using the rules in (3.9), but $\mathbf{u}_{a}$ is replaced by the DG velocity

$$
\begin{gathered}
\mathbf{u}_{a}^{D G}=-\lambda_{t} \mathbf{K} \nabla p_{h} \quad \text { in } E, \\
\mathbf{u}_{a}^{D G} \cdot \mathbf{n}=-\left\langle\lambda_{t}\left(S_{h}\right) \mathbf{K} \nabla p_{h} \cdot \mathbf{n}\right\rangle+\frac{r^{2} \sigma_{\gamma}}{h_{\gamma}}\left[p_{h}\right] \quad \text { on } \gamma \in \Gamma_{h} .
\end{gathered}
$$

With the boundary conditions, we have $\mathbf{u}_{a}^{D G} \cdot \mathbf{n}=0$ on $\Gamma_{h, N}$.

Since the proposed schemes employ the discontinuous finite element spaces to approximate the saturations, it allows that the approximate saturations may be discontinuous at the element interfaces although they are continuous within each element.

Let $p$ and $S$ be the solutions of the system of equations (2.4) and (2.5), which satisfy the regularity requirements: $p \in L^{2}\left(J ; H^{s}(\Omega)\right), S \in L^{\infty}\left(J ; L^{2}(\Omega)\right) \cap L^{2}\left(J ; H^{s}\left(\mathcal{E}_{h}^{0}\right)\right)$, $\frac{\partial S}{\partial t} \in L^{2}\left(J ; H^{s-1}(\Omega)\right)$, and $S^{0} \in H^{s-1}(\Omega)$, where $s>\frac{3}{2}$. Moreover, we assume that $p_{c}(S), \mathbf{u}_{a}$, and $\mathbf{u}_{c}$ are continuous in the entire domain, and $\nabla p$ and $\nabla S$ are essentially bounded in $\mathcal{E}_{h}^{0}$. For $t \in J$, the following equalities hold:

$$
\begin{aligned}
B_{p}(p, \varphi ; S) & =L_{p}(\varphi ; S), & \varphi & \in H^{s}\left(\mathcal{E}_{h}\right), \\
\left(\phi \frac{\partial S}{\partial t}, \varphi\right)+B_{S}(S, \varphi ; p) & =L_{S}(\varphi), & \varphi & \in H^{s}\left(\mathcal{E}_{h}\right) .
\end{aligned}
$$

Furthermore, for $\lambda_{c}$ defined by (3.8), we know that $\lambda_{c}<0$ and make the following assumption.

(H5) $\lambda_{c}$ is uniformly bounded both below and above, and $\lambda_{c}$ is also assumed to be Lipschitz continuous with respect to the saturation $S$; that is, $0<\lambda_{c}^{b} \leqslant\left|\lambda_{c}\right| \leqslant$ $\lambda_{c}^{a}, \quad\left|\lambda_{c}\left(S_{1}\right)-\lambda_{c}\left(S_{2}\right)\right| \leqslant L_{c}\left|S_{1}-S_{2}\right|$.

Define a function of saturation as

$$
\zeta(S):=\frac{\lambda_{n}(S)^{1 / 2}\left(M_{c}-p_{c}^{\prime}(S)\right)}{\lambda_{t}(S)^{1 / 2}\left|M_{c} p_{c}^{\prime}(S)\right|^{1 / 2}}
$$

where $M_{c}$ is a positive constant. It is noted that $\zeta(S) \geqslant 0$ from the physical meanings of $\lambda_{n}(S), \lambda_{t}(S)$, and $p_{c}^{\prime}(S)$. Furthermore, we assume the following.

(H6) There exist positive constants $M_{c}$ and $\delta$ such that $\max _{\mathcal{E}_{h} \times J} \zeta\left(S_{h}\right) \leqslant \delta<2$. For the sake of simplification, we choose $M_{c}=1$ in the theoretical analysis of this paper, but for the cases $M_{c} \neq 1$, the results can be obtained by making very small modifications only. 
For the boundary and initial data, we assume that $p_{B} \in L^{2}\left(J ; H^{1 / 2}\left(\Gamma_{D}\right)\right) \cap L^{\infty}(J \times$ $\left.\Gamma_{D}\right), S_{B} \in L^{\infty}\left(J ; H^{1 / 2}\left(\Gamma_{D}\right)\right) \cap L^{\infty}\left(J \times \Gamma_{D}\right), q_{w} \in L^{2}\left(J ; L^{2}(\Omega)\right), q_{n} \in L^{2}\left(J ; L^{2}(\Omega)\right)$. It is apparent that $q_{t} \in L^{2}\left(J ; L^{2}(\Omega)\right)$.

Throughout the paper, we use $C$ to indicate a generic positive constant independent of $h, r$, and use $\epsilon$ to indicate a small positive constant. The values of $C$ and $\epsilon$ are probably different in different occurrences.

4. Stability of discrete solutions. We now establish the stability of numerical solutions, which gives a priori estimates for the discrete pressure and saturation. For the purpose of theoretical analysis, we assume that there exist two constants $\theta_{0}>0, \theta_{1} \geqslant 0$ such that

$$
\theta_{0} J_{0}^{\sigma}\left(S_{h}, S_{h}\right)-\theta_{1} \leqslant-J_{0}^{\sigma}\left(p_{c}\left(S_{h}\right), S_{h}\right) .
$$

THEOREM 4.1. If the penalty parameter $\sigma_{0}$ is chosen to be sufficiently large and $h \leqslant h_{0}$, then we have

$$
\begin{gathered}
\left\|S_{h}\right\|_{L^{\infty}\left(J ; L^{2}\right)}^{2}+\left\|\boldsymbol{K}^{1 / 2} \nabla p_{h}\right\|_{L^{2}\left(J ; L^{2}\right)}^{2}+\left\|\boldsymbol{K}^{1 / 2} \nabla S_{h}\right\|_{L^{2}\left(J ; L^{2}\right)}^{2}+\int_{0}^{T} J_{0, D}^{\sigma}\left(S_{h}, S_{h}\right) \\
+\int_{0}^{T} J_{0, D}^{\sigma}\left(p_{h}, p_{h}\right) \leqslant C\left\|S^{0}\right\|_{0}^{2}+C \int_{0}^{T} J_{D}^{\sigma}\left(p_{B}, p_{B}\right)+C \int_{0}^{T} J_{D}^{\sigma}\left(S_{B}, S_{B}\right) \\
+C\left\|q_{w}\right\|_{L^{2}\left(J ; L^{2}\right)}^{2}+C\left\|q_{n}\right\|_{L^{2}\left(J ; L^{2}\right)}^{2}+C\left(p_{c m a x}^{2}+\theta_{1}\right),
\end{gathered}
$$

where $p_{\text {cmax }}$ is the upper bound of $p_{c}$.

Proof. Choosing $\varphi=p_{h}-S_{h}$ in (3.12), we obtain

$$
B_{p}\left(p_{h}, p_{h}-S_{h} ; S_{h}\right)=L_{p}\left(p_{h}-S_{h} ; S_{h}\right) .
$$

Furthermore, letting $\varphi=S_{h}$ in (3.13) gives us

$$
\left(\phi \frac{\partial S_{h}}{\partial t}, S_{h}\right)+B_{S}\left(S_{h}, S_{h} ; p_{h}\right)=L_{S}\left(S_{h}\right)
$$

We now combine (4.2) and (4.3), and obtain a coupled equation

$$
\begin{aligned}
(\phi & \left.\frac{\partial S_{h}}{\partial t}, S_{h}\right)+\sum_{E \in \mathcal{E}_{h}} \int_{E} \lambda_{t}\left(S_{h}\right) \mathbf{K} \nabla p_{h} \cdot \nabla p_{h}-\sum_{E \in \mathcal{E}_{h}} \int_{E} \lambda_{c}\left(S_{h}\right) \mathbf{K} \nabla S_{h} \cdot \nabla S_{h} \\
& +J_{0, D}^{\sigma}\left(p_{h}, p_{h}\right)-(\omega+1) J_{0}^{\sigma}\left(p_{c}\left(S_{h}\right), S_{h}\right)+J_{D}^{\sigma}\left(S_{h}, S_{h}\right) \\
= & \sum_{E \in \mathcal{E}_{h}} \int_{E}\left(\lambda_{n}\left(S_{h}\right)-\lambda_{c}\left(S_{h}\right)\right) \mathbf{K} \nabla p_{h} \cdot \nabla S_{h} \\
& +\left(1-s_{\text {form }}\right) \sum_{\gamma \in \Gamma_{h}} \int_{\gamma}\left\langle\lambda_{t}\left(S_{h}\right) \mathbf{K} \nabla p_{h} \cdot \mathbf{n}_{\gamma}\right\rangle\left[p_{h}\right] \\
& +\left(1-s_{\text {form }}\right) \sum_{\gamma \in \Gamma_{h, D}} \int_{\gamma}\left(\lambda_{t}\left(S_{B}\right) \mathbf{K} \nabla p_{h} \cdot \mathbf{n}_{\gamma}\right) p_{h}+J_{D}^{\sigma}\left(p_{B}, p_{h}\right) \\
& +\int_{\Omega} q_{t} p_{h}+s_{\text {form }} \sum_{\gamma \in \Gamma_{h, D}} \int_{\gamma} \lambda_{t}\left(S_{B}\right) \mathbf{K} \nabla p_{h} \cdot \mathbf{n}_{\gamma} p_{B} \\
& +\sum_{\gamma \in \Gamma_{h}} \int_{\gamma}\left(\left\langle\lambda_{c}\left(S_{h}\right) \mathbf{K} \nabla S_{h} \cdot \mathbf{n}_{\gamma}\right\rangle+s_{\text {form }} f_{n}\left(S_{h}^{*}\right)\left\langle\lambda_{t}\left(S_{h}\right) \mathbf{K} \nabla S_{h} \cdot \mathbf{n}_{\gamma}\right\rangle\right)\left[p_{h}\right]
\end{aligned}
$$

Copyright $@$ by SIAM. Unauthorized reproduction of this article is prohibited. 


$$
\begin{aligned}
& +\sum_{\gamma \in \Gamma_{h, D}} \int_{\gamma}\left(\lambda_{c}\left(S_{B}\right)+s_{\text {form }} \lambda_{n}\left(S_{B}\right)\right) \mathbf{K} \nabla S_{h} \cdot \mathbf{n}_{\gamma} p_{h} \\
& -\sum_{\gamma \in \Gamma_{h}} \int_{\gamma} f_{n}\left(S_{h}^{*}\right)\left\langle\lambda_{t}\left(S_{h}\right) \mathbf{K} \nabla p_{h} \cdot \mathbf{n}_{\gamma}\right\rangle\left[S_{h}\right] \\
& -\sum_{\gamma \in \Gamma_{h, D}} \int_{\gamma} \lambda_{n}\left(S_{B}\right) \mathbf{K} \nabla p_{h} \cdot \mathbf{n}_{\gamma} S_{h} \\
& -\sum_{\gamma \in \Gamma_{h}} \int_{\gamma}\left\langle\lambda_{c}\left(S_{h}\right) \mathbf{K} \nabla S_{h} \cdot \mathbf{n}_{\gamma}\right\rangle\left[S_{h}\right]+J_{D}^{\sigma}\left(S_{B}, S_{h}\right) \\
& -\sum_{\gamma \in \Gamma_{h, D}} \int_{\gamma} \lambda_{c}\left(S_{B}\right) \mathbf{K} \nabla S_{h} \cdot \mathbf{n}_{\gamma} S_{h}-s_{\text {form }} \sum_{\gamma \in \Gamma_{h, D}} \int_{\gamma} \lambda_{n}\left(S_{B}\right) \mathbf{K} \nabla S_{h} \cdot \mathbf{n}_{\gamma} p_{B} \\
& -s_{\text {form }} \sum_{\gamma \in \Gamma_{h}} \int_{\gamma}\left\langle\lambda_{n}\left(S_{h}\right) \mathbf{K} \nabla\left(p_{h}-S_{h}\right) \cdot \mathbf{n}_{\gamma}\right\rangle\left[p_{c}\left(S_{h}\right)\right] \\
& -J_{0}^{\sigma}\left(p_{c}\left(S_{h}\right), p_{h}\right)-\int_{\Omega} q_{n} S_{h} .
\end{aligned}
$$

First of all, we estimate the terms on the right-hand side of (4.4). The first term is bounded by using assumption (H6) and the Cauchy-Schwarz inequality as

$$
\begin{aligned}
& \sum_{E \in \mathcal{E}_{h}} \int_{E}\left(\lambda_{n}\left(S_{h}\right)-\lambda_{c}\left(S_{h}\right)\right) \mathbf{K} \nabla p_{h} \cdot \nabla S_{h} \\
& \quad=\sum_{E \in \mathcal{E}_{h}} \int_{E} \zeta\left(S_{h}\right) \lambda_{t}\left(S_{h}\right)^{1 / 2}\left|\lambda_{c}\left(S_{h}\right)\right|^{1 / 2} \mathbf{K} \nabla p_{h} \cdot \nabla S_{h} \\
& \quad \leqslant \frac{\delta}{2}\left(\left\|\left(\lambda_{t}\left(S_{h}\right) \mathbf{K}\right)^{1 / 2} \nabla p_{h}\right\|_{0}^{2}+\left\|\left(\left|\lambda_{c}\left(S_{h}\right)\right| \mathbf{K}\right)^{1 / 2} \nabla S_{h}\right\|_{0}^{2}\right),
\end{aligned}
$$

where $\delta \in(0,2)$. We now estimate the rest of the terms of the right-hand side in (4.4). The second and third terms on the right-hand side in (4.4) can be bounded as

$$
\begin{aligned}
& \left(1-s_{\text {form }}\right) \sum_{\gamma \in \Gamma_{h}} \int_{\gamma}\left\langle\lambda_{t}\left(S_{h}\right) \mathbf{K} \nabla p_{h} \cdot \mathbf{n}_{\gamma}\right\rangle\left[p_{h}\right] \\
& \quad+\left(1-s_{\text {form }}\right) \sum_{\gamma \in \Gamma_{h, D}} \int_{\gamma}\left(\lambda_{t}\left(S_{B}\right) \mathbf{K} \nabla p_{h} \cdot \mathbf{n}_{\gamma}\right) p_{h} \\
& \leqslant \sum_{E \in \mathcal{E}_{h}} \frac{h_{E}}{C r^{2}}\left\|\mathbf{K}^{1 / 2} \nabla p_{h} \cdot \mathbf{n}_{\partial E}\right\|_{0, \partial E}^{2}+\sum_{\gamma \in \Gamma_{h} \cup \Gamma_{h, D}} \frac{C r^{2}}{h_{\gamma}}\left\|\left[p_{h}\right]\right\|_{0, \gamma}^{2} \\
& \leqslant \epsilon_{1}\left\|\mathbf{K}^{1 / 2} \nabla p_{h}\right\|_{0}^{2}+\epsilon_{2} J_{0, D}^{\sigma}\left(p_{h}, p_{h}\right),
\end{aligned}
$$

where the penalty parameter $\sigma_{0}$ is chosen to be sufficiently large. The fourth term is bounded trivially,

$$
J_{D}^{\sigma}\left(p_{B}, p_{h}\right) \leqslant C J_{D}^{\sigma}\left(p_{B}, p_{B}\right)+\epsilon_{3} J_{D}^{\sigma}\left(p_{h}, p_{h}\right) \leqslant C J_{D}^{\sigma}\left(p_{B}, p_{B}\right)+\epsilon_{3} J_{0, D}^{\sigma}\left(p_{h}, p_{h}\right) .
$$

For the fifth term, we use Lemma 3.2 to obtain

$$
\begin{aligned}
\int_{\Omega} q_{t} p_{h} & \leqslant\left\|q_{t}\right\|_{0, \Omega}\left\|p_{h}\right\|_{0} \leqslant C\left\|q_{t}\right\|_{0, \Omega}\left(\left\|\nabla p_{h}\right\|_{0}^{2}+J_{0, D}^{\sigma}\left(p_{h}, p_{h}\right)\right)^{1 / 2} \\
& \leqslant C\left\|q_{t}\right\|_{0, \Omega}^{2}+\epsilon_{4}\left\|\mathbf{K}^{1 / 2} \nabla p_{h}\right\|_{0}^{2}+\epsilon_{5} J_{0, D}^{\sigma}\left(p_{h}, p_{h}\right) .
\end{aligned}
$$

Copyright $\odot$ by SIAM. Unauthorized reproduction of this article is prohibited. 
Similarly to the boundedness of the second term, the sixth term is estimated by

$$
s_{\text {form }} \sum_{\gamma \in \Gamma_{h, D}} \int_{\gamma} \lambda_{t}\left(S_{B}\right) \mathbf{K} \nabla p_{h} \cdot \mathbf{n}_{\gamma} p_{B} \leqslant \epsilon_{6}\left\|\mathbf{K}^{1 / 2} \nabla p_{h}\right\|_{0}^{2}+C J_{D}^{\sigma}\left(p_{B}, p_{B}\right) .
$$

The seventh and eighth terms are bounded similarly to the second term as

$$
\begin{aligned}
& \sum_{\gamma \in \Gamma_{h}} \int_{\gamma}\left(\left\langle\lambda_{c}\left(S_{h}\right) \mathbf{K} \nabla S_{h} \cdot \mathbf{n}_{\gamma}\right\rangle+s_{\text {form }} f_{n}\left(S_{h}^{*}\right)\left\langle\lambda_{t}\left(S_{h}\right) \mathbf{K} \nabla S_{h} \cdot \mathbf{n}_{\gamma}\right\rangle\right)\left[p_{h}\right] \\
& \quad+\sum_{\gamma \in \Gamma_{h, D}} \int_{\gamma}\left(\lambda_{c}\left(S_{B}\right)+s_{\text {form }} \lambda_{n}\left(S_{B}\right)\right) \mathbf{K} \nabla S_{h} \cdot \mathbf{n}_{\gamma} p_{h} \\
& \leqslant \frac{C}{\sigma_{0}} \sum_{E \in \mathcal{E}_{h}} \frac{h_{E}}{r^{2}}\left\|\mathbf{K}^{1 / 2} \nabla S_{h} \cdot \mathbf{n}_{\gamma}\right\|_{0, \gamma}^{2}+\epsilon \sum_{\gamma \in \Gamma_{h} \cup \Gamma_{h, D}} \frac{\sigma_{\gamma} r^{2}}{h_{\gamma}}\left\|\left[p_{h}\right]\right\|_{0, \gamma}^{2} \\
& \leqslant \varepsilon_{7}\left\|\mathbf{K}^{1 / 2} \nabla S_{h}\right\|_{0}^{2}+\epsilon_{8} J_{0, D}^{\sigma}\left(p_{h}, p_{h}\right) .
\end{aligned}
$$

The ninth and tenth terms on the right-hand side of (4.4) are bounded as

$$
\begin{aligned}
-\sum_{\gamma \in \Gamma_{h}} \int_{\gamma} f_{n}\left(S_{h}^{*}\right)\left\langle\lambda_{t}\left(S_{h}\right) \mathbf{K} \nabla p_{h} \cdot \mathbf{n}_{\gamma}\right\rangle\left[S_{h}\right]+ & \sum_{\gamma \in \Gamma_{h, D}} \int_{\gamma}\left(\lambda_{n}\left(S_{B}\right) \mathbf{K} \nabla p_{h} \cdot \mathbf{n}_{\gamma}\right) S_{h} \\
& \leqslant \epsilon_{9}\left\|\mathbf{K}^{1 / 2} \nabla p_{h}\right\|_{0}^{2}+\epsilon_{10} J_{0, D}^{\sigma}\left(S_{h}, S_{h}\right),
\end{aligned}
$$

where we need to choose suitable penalty parameters to estimate this bound. The eleventh and thirteenth terms are bounded as

$$
\begin{aligned}
-\sum_{\gamma \in \Gamma_{h}} \int_{\gamma}\left\langle\lambda_{c}\left(S_{h}\right) \mathbf{K} \nabla S_{h} \cdot \mathbf{n}_{\gamma}\right\rangle\left[S_{h}\right]-\sum_{\gamma \in \Gamma_{h, D}} & \int_{\gamma} \lambda_{c}\left(S_{B}\right) \mathbf{K} \nabla S_{h} \cdot \mathbf{n}_{\gamma} S_{h} \\
& \leqslant \epsilon_{12}\left\|\mathbf{K}^{1 / 2} \nabla S_{h}\right\|_{0}^{2}+\epsilon_{11} J_{0, D}^{\sigma}\left(S_{h}, S_{h}\right) .
\end{aligned}
$$

By the definition of $J_{D}^{\sigma}$, we bound the twelfth term

$$
J_{D}^{\sigma}\left(S_{B}, S_{h}\right) \leqslant C J_{D}^{\sigma}\left(S_{B}, S_{B}\right)+\epsilon_{13} J_{0, D}^{\sigma}\left(S_{h}, S_{h}\right) .
$$

The fourteenth term is estimated like the seventh term,

$$
-s_{\text {form }} \sum_{\gamma \in \Gamma_{h, D}} \int_{\gamma}\left\langle\lambda_{n}\left(S_{B}\right) \mathbf{K} \nabla S_{h} \cdot \mathbf{n}_{\gamma}\right\rangle p_{B} \leqslant C J_{D}^{\sigma}\left(p_{B}, p_{B}\right)+\epsilon_{14}\left\|\mathbf{K}^{1 / 2} \nabla S_{h}\right\|_{0}^{2} .
$$

The fifteenth term is estimated like the seventh term,

$$
\begin{aligned}
-s_{\text {form }} \sum_{\gamma \in \Gamma_{h}} \int_{\gamma}\left\langle\lambda _ { h } ( S _ { h } ) \mathbf { K } \nabla \left( p_{h}-\right.\right. & \left.\left.S_{h}\right) \cdot \mathbf{n}_{\gamma}\right\rangle\left[p_{c}\left(S_{h}\right)\right] \\
& \leqslant C p_{c m a x}^{2}+\epsilon_{15}\left\|\mathbf{K}^{1 / 2} \nabla p_{h}\right\|_{0}^{2}+\epsilon_{16}\left\|\mathbf{K}^{1 / 2} \nabla S_{h}\right\|_{0}^{2} .
\end{aligned}
$$

The sixteenth term is bounded as $J_{0}^{\sigma}\left(p_{c}\left(S_{h}\right), p_{h}\right) \leqslant C p_{\text {cmax }}^{2}+\frac{1}{2} J_{0}^{\sigma}\left(p_{h}, p_{h}\right)$. The last term is bounded by $\frac{1}{2}\left\|q_{n}\right\|_{0, \Omega}^{2}+\frac{1}{2}\left\|S_{h}\right\|_{0}^{2}$. 
Noticing that $\left(\phi \frac{\partial S_{h}}{\partial t}, S_{h}\right)=\frac{1}{2} \frac{d}{d t}\left\|\phi^{1 / 2} S_{h}\right\|_{0}^{2}$, we move (4.5) to the left-hand side of (4.4), combine the above estimates on the right-hand side of (4.4), and obtain

$$
\begin{aligned}
\frac{1}{2} \frac{d}{d t} & \left\|\phi^{\frac{1}{2}} S_{h}\right\|_{0}^{2}+c_{0}\left\|\mathbf{K}^{1 / 2} \nabla p_{h}\right\|_{0}^{2} \\
& +c_{1}\left\|\mathbf{K}^{1 / 2} \nabla S_{h}\right\|_{0}^{2}+\min \left(\theta_{0}, 1\right) J_{0, D}^{\sigma}\left(S_{h}, S_{h}\right)+\frac{1}{2} J_{0, D}^{\sigma}\left(p_{h}, p_{h}\right) \\
\leqslant & \frac{1}{2}\left\|S_{h}\right\|_{0}^{2}+\left(\varepsilon_{1}+\varepsilon_{4}+\varepsilon_{6}+\varepsilon_{9}+\varepsilon_{15}\right)\left\|\mathbf{K}^{1 / 2} \nabla p_{h}\right\|_{0}^{2} \\
& +\left(\varepsilon_{7}+\varepsilon_{12}+\varepsilon_{14}+\varepsilon_{16}\right)\left\|\mathbf{K}^{1 / 2} \nabla S_{h}\right\|_{0}^{2}+\left(\varepsilon_{2}+\varepsilon_{3}+\varepsilon_{5}+\varepsilon_{8}\right) J_{0, D}^{\sigma}\left(p_{h}, p_{h}\right) \\
& +\left(\varepsilon_{10}+\varepsilon_{11}+\varepsilon_{13}\right) J_{0, D}^{\sigma}\left(S_{h}, S_{h}\right)+C J_{D}^{\sigma}\left(p_{B}, p_{B}\right) \\
& +C J_{D}^{\sigma}\left(S_{B}, S_{B}\right)+C\left(\left\|q_{w}\right\|_{0, \Omega}^{2}+\left\|q_{n}\right\|_{0, \Omega}^{2}\right)+C\left(p_{c m a x}^{2}+\theta_{1}\right),
\end{aligned}
$$

where $c_{0}, c_{1}$ are two positive constants depending on $\lambda_{t}, \lambda_{c}, \delta$. Furthermore, using the boundedness of $\phi, \lambda_{t}$, and $\lambda_{c}$, and suitably adjusting the parameters $\varepsilon_{i}>0$ such that

$$
\begin{aligned}
\varepsilon_{1}+\varepsilon_{4}+\varepsilon_{6}+\varepsilon_{9}+\varepsilon_{15} & =\frac{1}{2} c_{0}, & \varepsilon_{7}+\varepsilon_{12}+\varepsilon_{14}+\varepsilon_{16} & =\frac{1}{2} c_{1}, \\
\varepsilon_{2}+\varepsilon_{3}+\varepsilon_{5}+\varepsilon_{8} & =\frac{1}{4}, & \varepsilon_{10}+\varepsilon_{11}+\varepsilon_{13} & =\frac{1}{2} \min \left(\theta_{0}, 1\right),
\end{aligned}
$$

we get

$$
\begin{aligned}
\frac{d}{d t} \| & S_{h}\left\|_{0}^{2}+\right\| \mathbf{K}^{1 / 2} \nabla p_{h}\left\|_{0}^{2}+\right\| \mathbf{K}^{1 / 2} \nabla S_{h} \|_{0}^{2}+J_{0, D}^{\sigma}\left(S_{h}, S_{h}\right)+J_{0, D}^{\sigma}\left(p_{h}, p_{h}\right) \\
\leqslant & C\left\|S_{h}\right\|_{0}^{2}+C J_{D}^{\sigma}\left(p_{B}, p_{B}\right)+C J_{D}^{\sigma}\left(S_{B}, S_{B}\right) \\
& +C\left(\left\|q_{w}\right\|_{0, \Omega}^{2}+\left\|q_{n}\right\|_{0, \Omega}^{2}\right)+C\left(p_{\text {cmax }}^{2}+\theta_{1}\right) .
\end{aligned}
$$

Finally, taking into account the fact $\left\|S_{h}^{0}\right\|_{0} \leqslant\left\|S^{0}\right\|_{0, \Omega}$, the inequality (4.1) can be obtained by Gronwall's inequality.

Remark. From Theorem 4.1 and Lemma 3.2, we know that $p_{h} \in L^{2}\left(J ; H^{1}\left(\mathcal{E}_{h}\right)\right)$ and $S_{h} \in L^{\infty}\left(J ; L^{2}(\Omega)\right) \bigcap L^{2}\left(J ; H^{1}\left(\mathcal{E}_{h}\right)\right)$, where $J=(0, T]$.

\section{Error estimates.}

5.1. Approximation results. In order to derive the error estimates, we first recall the following well-known $h p$-approximation results, which can be proved using the techniques in [3,4]. Let $E \in \mathcal{E}_{h}$ and $v \in H^{s}(E)$, then there exists a constant $C$ independent of $v, r$, and $h_{E}$, and a sequence of $z_{r}^{h_{E}} \in \mathbb{P}_{r}(E), r \geqslant 1$, such that

$$
\begin{aligned}
\left\|v-z_{r}^{h_{E}}\right\|_{q, E} \leqslant C \frac{h_{E}^{\mu-q}}{r^{s-q}}\|v\|_{s, E}, & 0 \leqslant q<\mu, \\
\left\|v-z_{r}^{h_{E}}\right\|_{q, \partial E} \leqslant C \frac{h_{E}^{\mu-q-\frac{1}{2}}}{r^{s-q-\frac{1}{2}}}\|v\|_{s, E}, & 0 \leqslant q<\mu-\frac{1}{2},
\end{aligned}
$$

where $\mu=\min (r+1, s)$ and $h_{E}$ is the diameter of $E$.

5.2. Error estimates. Let $\widehat{p}$ and $\widehat{S}$ be the interpolants of the pressure $p$ and saturation $S$ in $\mathcal{D}_{r}\left(\mathcal{E}_{h}\right)$ such that the $h p$-approximation results (5.1) and (5.2) hold.

Copyright $@$ by SIAM. Unauthorized reproduction of this article is prohibited. 
Define

$$
\begin{array}{ccc}
E_{p}=p-p_{h}, & E_{p}^{I}=\widehat{p}-p, & E_{p}^{A}=E_{p}^{I}+E_{p}=\widehat{p}-p_{h}, \\
E_{S}=S-S_{h}, & E_{S}^{I}=\widehat{S}-S, \quad E_{S}^{A}=E_{S}^{I}+E_{S}=\widehat{S}-S_{h} .
\end{array}
$$

Moreover, it is assumed that for $h \leqslant h_{0}$, there exist positive constants $a_{0}, a_{1}$ such that

$$
\begin{aligned}
& a_{0} J_{0}^{\sigma}\left(E_{S}^{A}, E_{S}^{A}\right) \leqslant-J_{0}^{\sigma}\left(p_{c}(\widehat{S})-p_{c}\left(S_{h}\right), E_{S}^{A}\right)+C \frac{h^{\min (2 r, 2 s-2)}}{r^{2 s-3}}, \\
& J_{0}^{\sigma}\left(p_{c}(\widehat{S})-p_{c}\left(S_{h}\right), p_{c}(\widehat{S})-p_{c}\left(S_{h}\right)\right) \leqslant a_{1} J_{0}^{\sigma}\left(E_{S}^{A}, E_{S}^{A}\right)+C \frac{h^{\min (2 r, 2 s-2)}}{r^{2 s-3}} .
\end{aligned}
$$

Subtracting the pressure DG scheme (3.12) from the weak formulation (3.15), we can obtain

$$
\begin{aligned}
\sum_{E \in \mathcal{E}_{h}} & \int_{E} \lambda_{t}\left(S_{h}\right) \mathbf{K} \nabla E_{p}^{A} \cdot \nabla \varphi+J_{0, D}^{\sigma}\left(E_{p}^{A}, \varphi\right)-J_{0, D}^{\sigma}\left(E_{p}^{I}, \varphi\right) \\
& -\sum_{E \in \mathcal{E}_{h}} \int_{E} \lambda_{t}\left(S_{h}\right) \mathbf{K} \nabla E_{p}^{I} \cdot \nabla \varphi+\sum_{E \in \mathcal{E}_{h}} \int_{E}\left(\lambda_{t}(S)-\lambda_{t}\left(S_{h}\right)\right) \mathbf{K} \nabla p \cdot \nabla \varphi \\
& -\sum_{\gamma \in \Gamma_{h}} \int_{\gamma}\left\langle\left(\lambda_{t}(S)-\lambda_{t}\left(S_{h}\right)\right) \mathbf{K} \nabla p \cdot \mathbf{n}_{\gamma}\right\rangle[\varphi]-\sum_{\gamma \in \Gamma_{h}} \int_{\gamma}\left\langle\lambda_{t}\left(S_{h}\right) \mathbf{K} \nabla E_{p} \cdot \mathbf{n}_{\gamma}\right\rangle[\varphi] \\
& -\sum_{\gamma \in \Gamma_{h, D}} \int_{\gamma}\left(\lambda_{t}\left(S_{B}\right) \mathbf{K} \nabla E_{p} \cdot \mathbf{n}_{\gamma}\right) \varphi+s_{\text {form }} \sum_{\gamma \in \Gamma_{h}} \int_{\gamma}\left\langle\lambda_{t}\left(S_{h}\right) \mathbf{K} \nabla \varphi \cdot \mathbf{n}_{\gamma}\right\rangle\left[E_{p}\right] \\
& +s_{\text {form }} \sum_{\gamma \in \Gamma_{h, D}} \int_{\gamma}\left(\lambda_{t}\left(S_{B}\right) \mathbf{K} \nabla \varphi \cdot \mathbf{n}_{\gamma}\right) E_{p} \\
= & L_{p}(\varphi ; S)-L_{p}\left(\varphi ; S_{h}\right), \quad \varphi \in \mathcal{D}_{r}\left(\mathcal{E}_{h}\right) .
\end{aligned}
$$

We note that the jumps $[p]$ are zero almost everywhere on the interior edges (or faces), and thus some terms vanish. Subtracting the saturation DG scheme (3.13) from the weak formulation (3.16), and letting $\varphi=E_{S}^{A}$, we can obtain

$$
\begin{aligned}
(\phi & \left.\frac{\partial E_{S}^{A}}{\partial t}, E_{S}^{A}\right)-\omega J_{0}^{\sigma}\left(p_{c}(\widehat{S})-p_{c}\left(S_{h}\right), E_{S}^{A}\right)+J_{D}^{\sigma}\left(E_{S}^{A}, E_{S}^{A}\right) \\
= & \left(\phi \frac{\partial E_{S}^{I}}{\partial t}, E_{S}^{A}\right)-\sum_{E \in \mathcal{E}_{h}} \int_{E}\left(\lambda_{w}(S)-\lambda_{w}\left(S_{h}\right)\right) \mathbf{K} \nabla p \cdot \nabla E_{S}^{A} \\
& -\sum_{E \in \mathcal{E}_{h}} \int_{E} \lambda_{w}\left(S_{h}\right) \mathbf{K} \nabla E_{p} \cdot \nabla E_{S}^{A}+\sum_{\gamma \in \Gamma_{h}} \int_{\gamma}\left(f_{w}\left(S^{*}\right)\left\langle\lambda_{t}(S) \mathbf{K} \nabla p \cdot \mathbf{n}_{\gamma}\right\rangle\right. \\
& +\sum_{\gamma \in \Gamma_{h}} \int_{\gamma} f_{w}\left(S_{h}^{*}\right)\left\langle\lambda_{t}\left(S_{h}\right) \mathbf{K} \nabla E_{p} \cdot \mathbf{n}_{\gamma}\right\rangle\left[E_{S}^{A}\right] \\
& \left.+\sum_{\gamma \in \Gamma_{h}, D} \int_{\gamma}\left(\lambda_{w}\left(S_{h}\right) \mathbf{K} \nabla p \cdot \mathbf{n}_{\gamma}\right\rangle\right)\left[E_{S}^{A}\right] \\
& -J_{0, D}^{\sigma}\left(p-p_{h}, E_{S}^{A}\right)+E_{D}^{\sigma}\left(E_{S}^{I}, E_{S}^{A}\right) \\
& -s_{\text {form }} \sum_{\gamma \in \Gamma_{h}} \int_{\gamma} f_{w}\left(S_{h}^{*}\right)\left\langle\lambda_{t}\left(S_{h}\right) \mathbf{K} \nabla E_{S}^{A} \cdot \mathbf{n}_{\gamma}\right\rangle\left[E_{p}\right] \\
& -s_{\text {form }} \sum_{\gamma \in \Gamma_{h, D}} \int_{\gamma}\left(\lambda_{w}\left(S_{B}\right) \mathbf{K} \nabla E_{S}^{A} \cdot \mathbf{n}_{\gamma}\right) E_{p}+\omega J_{0}^{\sigma}\left(p_{c}(S)-p_{c}(\widehat{S}), E_{S}^{A}\right)
\end{aligned}
$$

Copyright $@$ by SIAM. Unauthorized reproduction of this article is prohibited. 
In the conventional technique for the error analysis of two-phase flow, for example $[16,17]$, one first analyzes the pressure equation and the saturation equation separately, and then combines them together. This technique is conveniently carried out for the classical two-phase flow formulation; however, for our formulation, there is a great difficulty handling the broken gradients of saturation in the pressure equation. In order to overcome this difficulty, we introduce a coupling approach for the pressure and saturation equations, and then obtain a coupled error equation, which will conveniently yield the required error estimates. Choosing $\varphi=E_{p}^{A}-E_{S}^{A}$ in (5.3), and then adding the obtained equation to (5.4), after some simplifications, we obtain a coupled error equation

$$
\begin{aligned}
(\phi & \left.\frac{\partial E_{S}^{A}}{\partial t}, E_{S}^{A}\right)-(\omega+1) J_{0}^{\sigma}\left(p_{c}(\widehat{S})-p_{c}\left(S_{h}\right), E_{S}^{A}\right)+J_{D}^{\sigma}\left(E_{S}^{A}, E_{S}^{A}\right) \\
& +\sum_{E \in \mathcal{E}_{h}} \int_{E} \lambda_{t}\left(S_{h}\right) \mathbf{K} \nabla E_{p}^{A} \cdot \nabla E_{p}^{A}+J_{0, D}^{\sigma}\left(E_{p}^{A}, E_{p}^{A}\right) \\
& -\sum_{E \in \mathcal{E}_{h}} \int_{E} \lambda_{c}\left(S_{h}\right) \mathbf{K} \nabla E_{S}^{A} \cdot \nabla E_{S}^{A} \\
= & \sum_{E \in \mathcal{E}_{h}} \int_{E}\left(\lambda_{n}\left(S_{h}\right)-\lambda_{c}\left(S_{h}\right)\right) \mathbf{K} \nabla E_{p}^{A} \cdot \nabla E_{S}^{A}+G_{p}+G_{S}
\end{aligned}
$$

where

$$
\begin{aligned}
G_{p}:= & \sum_{E \in \mathcal{E}_{h}} \int_{E} \lambda_{t}\left(S_{h}\right) \mathbf{K} \nabla E_{p}^{I} \cdot \nabla E_{p}^{A}-\sum_{E \in \mathcal{E}_{h}} \int_{E}\left(\lambda_{t}(S)-\lambda_{t}\left(S_{h}\right)\right) \mathbf{K} \nabla p \cdot \nabla E_{p}^{A} \\
& +\sum_{\gamma \in \Gamma_{h}} \int_{\gamma}\left\langle\left(\lambda_{t}(S)-\lambda_{t}\left(S_{h}\right)\right) \mathbf{K} \nabla p \cdot \mathbf{n}_{\gamma}\right\rangle\left[E_{p}^{A}\right]+\sum_{\gamma \in \Gamma_{h}} \int_{\gamma}\left\langle\lambda_{t}\left(S_{h}\right) \mathbf{K} \nabla E_{p} \cdot \mathbf{n}_{\gamma}\right\rangle\left[E_{p}^{A}\right] \\
& +\sum_{\gamma \in \Gamma_{h, D}} \int_{\gamma}\left(\lambda_{t}\left(S_{B}\right) \mathbf{K} \nabla E_{p} \cdot \mathbf{n}_{\gamma}\right) E_{p}^{A}-s_{\text {form }} \sum_{\gamma \in \Gamma_{h}} \int_{\gamma}\left\langle\lambda_{t}\left(S_{h}\right) \mathbf{K} \nabla E_{p}^{A} \cdot \mathbf{n}_{\gamma}\right\rangle\left[E_{p}\right] \\
& -s_{\text {form }} \sum_{\gamma \in \Gamma_{h, D}} \int_{\gamma}\left(\lambda_{t}\left(S_{B}\right) \mathbf{K} \nabla E_{p}^{A} \cdot \mathbf{n}_{\gamma}\right) E_{p}+J_{0, D}^{\sigma}\left(E_{p}^{I}, E_{p}^{A}\right) \\
& +\sum_{E \in \mathcal{E}_{h}} \int_{E} \lambda_{c}\left(S_{h}\right) \mathbf{K} \nabla E_{S}^{I} \cdot \nabla E_{p}^{A}-\sum_{E \in \mathcal{E}_{h}} \int_{E}\left(\lambda_{c}(S)-\lambda_{c}\left(S_{h}\right)\right) \mathbf{K} \nabla S \cdot \nabla E_{p}^{A} \\
& +\sum_{\gamma \in \Gamma_{h}} \int_{\gamma}\left\langle\lambda_{c}\left(S_{h}\right) \mathbf{K} \nabla E_{S} \cdot \mathbf{n}_{\gamma}\right\rangle\left[E_{p}^{A}\right]+\sum_{\gamma \in \Gamma_{h}} \int_{\gamma}\left\langle\left(\lambda_{c}(S)-\lambda_{c}\left(S_{h}\right)\right) \mathbf{K} \nabla S \cdot \mathbf{n}_{\gamma}\right\rangle\left[E_{p}^{A}\right] \\
& +\sum_{\gamma \in \Gamma_{h, D}} \int_{\gamma} \lambda_{c}\left(S_{B}\right) \mathbf{K} \nabla E_{S} \cdot \mathbf{n}_{\gamma} E_{p}^{A}-J_{0}^{\sigma}\left(p_{c}(S)-p_{c}\left(S_{h}\right), E_{p}^{A}\right), \\
G_{S}= & -\sum_{E \in \mathcal{E}_{h}} \int_{E} \lambda_{n}\left(S_{h}\right) \mathbf{K} \nabla E_{p}^{I} \cdot \nabla E_{S}^{A}+\sum_{E \in \mathcal{E}_{h}} \int_{E}\left(\lambda_{n}(S)-\lambda_{n}\left(S_{h}\right)\right) \mathbf{K} \nabla p \cdot \nabla E_{S}^{A} \\
& -\sum_{\gamma \in \Gamma_{h}} \int_{\gamma}\left(f_{n}\left(S^{*}\right)\left\langle\lambda_{t}(S) \mathbf{K} \nabla p \cdot \mathbf{n}_{\gamma}\right\rangle-f_{n}\left(S_{h}^{*}\right)\left\langle\lambda_{t}\left(S_{h}\right) \mathbf{K} \nabla p \cdot \mathbf{n}_{\gamma}\right\rangle\right)\left[E_{S}^{A}\right] \\
& -\sum_{\gamma \in \Gamma_{h}} \int_{\gamma} f_{n}\left(S_{h}^{*}\right)\left\langle\lambda_{t}\left(S_{h}\right) \mathbf{K} \nabla E_{p} \cdot \mathbf{n}_{\gamma}\right\rangle\left[E_{S}^{A}\right]-\sum_{\gamma \in \Gamma_{h}, D} \int_{\gamma}\left(\lambda_{n}\left(S_{B}\right) \mathbf{K} \nabla E_{p} \cdot \mathbf{n}_{\gamma}\right) E_{S}^{A}
\end{aligned}
$$

Copyright (c) by SIAM. Unauthorized reproduction of this article is prohibited. 


$$
\begin{aligned}
& +s_{\text {form }} \sum_{\gamma \in \Gamma_{h}} \int_{\gamma} f_{n}\left(S_{h}^{*}\right)\left\langle\lambda_{t}\left(S_{h}\right) \mathbf{K} \nabla E_{S}^{A} \cdot \mathbf{n}_{\gamma}\right\rangle\left[E_{p}\right] \\
& +s_{\text {form }} \sum_{\gamma \in \Gamma_{h, D}} \int_{\gamma}\left(\lambda_{n}\left(S_{B}\right) \mathbf{K} \nabla E_{S}^{A} \cdot \mathbf{n}_{\gamma}\right) E_{p}-\sum_{E \in \mathcal{E}_{h}} \int_{E} \lambda_{c}\left(S_{h}\right) \mathbf{K} \nabla E_{S}^{I} \cdot \nabla E_{S}^{A} \\
& +\sum_{E \in \mathcal{E}_{h}} \int_{E}\left(\lambda_{c}(S)-\lambda_{c}\left(S_{h}\right)\right) \mathbf{K} \nabla S \cdot \nabla E_{S}^{A}-\sum_{\gamma \in \Gamma_{h}} \int_{\gamma}\left\langle\lambda_{c}\left(S_{h}\right) \mathbf{K} \nabla E_{S} \cdot \mathbf{n}_{\gamma}\right\rangle\left[E_{S}^{A}\right] \\
& -\sum_{\gamma \in \Gamma_{h}} \int_{\gamma}\left\langle\left(\lambda_{c}(S)-\lambda_{c}\left(S_{h}\right)\right) \mathbf{K} \nabla S \cdot \mathbf{n}_{\gamma}\right\rangle\left[E_{S}^{A}\right]-\sum_{\gamma \in \Gamma_{h}, D} \int_{\gamma} \lambda_{c}\left(S_{B}\right) \mathbf{K} \nabla E_{S} \cdot \mathbf{n}_{\gamma} E_{S}^{A} \\
& -s_{\text {form }} \sum_{\gamma \in \Gamma_{h}} \int_{\gamma}\left\langle\lambda_{n}\left(S_{h}\right) \mathbf{K} \nabla\left(E_{p}^{A}-E_{S}^{A}\right) \cdot \mathbf{n}_{\gamma}\right\rangle\left[p_{c}(S)-p_{c}\left(S_{h}\right)\right] \\
& +(\omega+1) J_{0}^{\sigma}\left(p_{c}(S)-p_{c}(\widehat{S}), E_{S}^{A}\right)+J_{D}^{\sigma}\left(E_{S}^{I}, E_{S}^{A}\right)+\left(\phi \frac{\partial E_{S}^{I}}{\partial t}, E_{S}^{A}\right) .
\end{aligned}
$$

For the first term on the right-hand side of (5.5), using the Cauchy-Schwarz inequality, we have

$$
\begin{aligned}
& \sum_{E \in \mathcal{E}_{h}} \int_{E}\left(\lambda_{n}\left(S_{h}\right)-\lambda_{c}\left(S_{h}\right)\right) \mathbf{K} \nabla E_{p}^{A} \cdot \nabla E_{S}^{A} \\
& \quad=\sum_{E \in \mathcal{E}_{h}} \int_{E} \zeta\left(S_{h}\right) \lambda_{t}\left(S_{h}\right)^{\frac{1}{2}}\left|\lambda_{c}\left(S_{h}\right)\right|^{\frac{1}{2}} \mathbf{K} \nabla E_{p}^{A} \cdot \nabla E_{S}^{A} \\
& \quad \leqslant \frac{\delta}{2}\left\|\left(\lambda_{t}\left(S_{h}\right) \mathbf{K}\right)^{\frac{1}{2}} \nabla E_{p}^{A}\right\|_{0}^{2}+\frac{\delta}{2}\left\|\left(\left|\lambda_{c}\left(S_{h}\right)\right| \mathbf{K}\right)^{1 / 2} \nabla E_{S}^{A}\right\|_{0}^{2},
\end{aligned}
$$

where we have used the assumption on $\zeta(S)$ and $\delta \in(0,2)$. We substitute (5.6) into (5.5) and obtain

$$
\begin{aligned}
& \left(\phi \frac{\partial E_{S}^{A}}{\partial t}, E_{S}^{A}\right)+(\omega+1) a_{0} J_{0}^{\sigma}\left(E_{S}^{A}, E_{S}^{A}\right)+J_{D}^{\sigma}\left(E_{S}^{A}, E_{S}^{A}\right)+J_{0, D}^{\sigma}\left(E_{p}^{A}, E_{p}^{A}\right) \\
& +\left(1-\frac{\delta}{2}\right)\left(\left\|\left(\lambda_{t}\left(S_{h}\right) \mathbf{K}\right)^{1 / 2} \nabla E_{p}^{A}\right\|_{0}^{2}+\left\|\left(\left|\lambda_{c}\left(S_{h}\right)\right| \mathbf{K}\right)^{1 / 2} \nabla E_{S}^{A}\right\|_{0}^{2}\right) \leqslant G_{p}+G_{S}+C \frac{h^{\min (2 r, 2 s-2)}}{r^{2 s-3}} .
\end{aligned}
$$

In addition, we can see that $\left(\phi \frac{\partial E_{S}^{A}}{\partial t}, E_{S}^{A}\right)=\frac{1}{2} \frac{d}{d t}\left\|\phi^{1 / 2} E_{S}^{A}\right\|_{0}^{2} \geqslant \frac{\phi^{b}}{2} \frac{d}{d t}\left\|E_{S}^{A}\right\|_{0}^{2}$ because of the boundedness of porosity. Furthermore, since $\lambda_{t}$ and $\left|\lambda_{c}\right|$ are bounded below, the inequality (5.7) can be changed into

$$
\begin{aligned}
\frac{\phi^{b}}{2} \frac{d}{d t}\left\|E_{S}^{A}\right\|_{0}^{2} & +(\omega+1) a_{0} J_{0}^{\sigma}\left(E_{S}^{A}, E_{S}^{A}\right)+J_{D}^{\sigma}\left(E_{S}^{A}, E_{S}^{A}\right)+\kappa_{1}\left\|\mathbf{K}^{1 / 2} \nabla E_{p}^{A}\right\|_{0}^{2} \\
& +\kappa_{2}\left\|\mathbf{K}^{1 / 2} \nabla E_{S}^{A}\right\|_{0}^{2}+J_{0, D}^{\sigma}\left(E_{p}^{A}, E_{p}^{A}\right) \leqslant G_{p}+G_{S}+C \frac{h^{\min (2 r, 2 s-2)}}{r^{2 s-3}}
\end{aligned}
$$

where $\kappa_{1}$ and $\kappa_{2}$ depend on $\delta, \lambda_{t}, \lambda_{c}$.

We estimate the two parts $G_{p}$ and $G_{S}$ in Appendixes A and B, respectively. Taking $\omega \geqslant \frac{a_{1}}{a_{0}}$ and substituting the estimates (A.1) and (B.1) of $G_{p}$ and $G_{S}$ into 
(5.8), we obtain

$$
\begin{aligned}
(5.9) \frac{d}{d t} \| & E_{S}^{A}\left\|_{0}^{2}+\kappa_{1}\right\| \mathbf{K}^{1 / 2} \nabla E_{p}^{A}\left\|_{0}^{2}+\kappa_{2}\right\| \mathbf{K}^{1 / 2} \nabla E_{S}^{A} \|_{0}^{2}+\frac{1}{2} J_{0, D}^{\sigma}\left(E_{p}^{A}, E_{p}^{A}\right) \\
& +a_{0} J_{0}^{\sigma}\left(E_{S}^{A}, E_{S}^{A}\right)+J_{D}^{\sigma}\left(E_{S}^{A}, E_{S}^{A}\right) \\
\leqslant & \left(\epsilon_{1}^{p}+\epsilon_{2}^{p}+\epsilon_{5}^{p}+\epsilon_{6}^{p}+\epsilon_{8}^{p}+\epsilon_{9}^{p}+\epsilon_{11}^{p}+\epsilon_{14}^{p}+\epsilon_{15}^{p}+\epsilon_{5}^{s}+\epsilon_{6}^{s}+\epsilon_{19}^{s}\right)\left\|\mathbf{K}^{1 / 2} \nabla E_{p}^{A}\right\|_{0}^{2} \\
& +\left(\epsilon_{3}^{p}+\epsilon_{4}^{p}+\epsilon_{7}^{p}+\epsilon_{10}^{p}+\epsilon_{12}^{p}+\epsilon_{13}^{p}+\epsilon_{16}^{p}+\epsilon_{18}^{p}+\epsilon_{20}^{p}+\epsilon_{8}^{s}+\epsilon_{11}^{s}\right) J_{0, D}^{\sigma}\left(E_{p}^{A}, E_{p}^{A}\right) \\
& +\left(\epsilon_{17}^{p}+\epsilon_{19}^{p}+\epsilon_{1}^{s}+\epsilon_{2}^{s}+\epsilon_{9}^{s}+\epsilon_{10}^{s}+\epsilon_{12}^{s}+\epsilon_{13}^{s}+\epsilon_{14}^{s}+\epsilon_{17}^{s}+\epsilon_{20}^{s}\right)\left\|\mathbf{K}^{1 / 2} \nabla E_{S}^{A}\right\|_{0}^{2} \\
& +\left(\epsilon_{3}^{s}+\epsilon_{4}^{s}+\epsilon_{7}^{s}+\epsilon_{15}^{s}+\epsilon_{16}^{s}+\epsilon_{18}^{s}+\epsilon_{21}^{s}+\epsilon_{22}^{s}\right) J_{0, D}^{\sigma}\left(E_{S}^{A}, E_{S}^{A}\right) \\
& +C\left\|E_{S}^{A}\right\|_{0}^{2}+C\left(\|p\|_{s}^{2}+\|S\|_{s}^{2}+\left\|S_{t}\right\|_{s-1}^{2}\right) \frac{h^{\min (2 r, 2 s-2)}}{r^{2 s-3}} .
\end{aligned}
$$

Choosing sufficiently small $\epsilon_{i}^{p}$ and $\epsilon_{i}^{s}$ such that

$$
\begin{aligned}
& \epsilon_{1}^{p}+\epsilon_{2}^{p}+\epsilon_{5}^{p}+\epsilon_{6}^{p}+\epsilon_{8}^{p}+\epsilon_{9}^{p}+\epsilon_{11}^{p}+\epsilon_{14}^{p}+\epsilon_{15}^{p}+\epsilon_{5}^{s}+\epsilon_{6}^{s}+\epsilon_{19}^{s}=\frac{\kappa_{1}}{2}, \\
& \epsilon_{3}^{p}+\epsilon_{4}^{p}+\epsilon_{7}^{p}+\epsilon_{10}^{p}+\epsilon_{12}^{p}+\epsilon_{13}^{p}+\epsilon_{16}^{p}+\epsilon_{18}^{p}+\epsilon_{20}^{p}+\epsilon_{8}^{s}+\epsilon_{11}^{s}=\frac{1}{4}, \\
& \epsilon_{17}^{p}+\epsilon_{19}^{p}+\epsilon_{1}^{s}+\epsilon_{2}^{s}+\epsilon_{9}^{s}+\epsilon_{10}^{s}+\epsilon_{12}^{s}+\epsilon_{13}^{s}+\epsilon_{14}^{s}+\epsilon_{17}^{s}+\epsilon_{20}^{s}=\frac{\kappa_{2}}{2}, \\
& \epsilon_{3}^{s}+\epsilon_{4}^{s}+\epsilon_{7}^{s}+\epsilon_{15}^{s}+\epsilon_{16}^{s}+\epsilon_{18}^{s}+\epsilon_{21}^{s}+\epsilon_{22}^{s}=\frac{1}{2} \min \left(a_{0}, 1\right),
\end{aligned}
$$

and taking into account $\left\|E_{S}^{A}\right\|_{0}(0)=0$, by Gronwall's inequality, we obtain

$$
\begin{aligned}
\left\|E_{S}^{A}\right\|_{0}^{2}(\tau)+ & \int_{0}^{T}\left\|\mathbf{K}^{1 / 2} \nabla E_{p}^{A}\right\|_{0}^{2}+\int_{0}^{T}\left\|\mathbf{K}^{1 / 2} \nabla E_{S}^{A}\right\|_{0}^{2} \\
& +\int_{0}^{T} J_{0, D}^{\sigma}\left(E_{p}^{A}, E_{p}^{A}\right)+\int_{0}^{T} J_{0, D}^{\sigma}\left(E_{S}^{A}, E_{S}^{A}\right) \\
\leqslant & C\left(\|p\|_{L^{2}\left(J ; H^{s}\right)}^{2}+\|S\|_{L^{2}\left(J ; H^{s}\right)}^{2}+\left\|S_{t}\right\|_{L^{2}\left(J ; H^{s-1}\right)}^{2}\right) \frac{h^{\min (2 r, 2 s-2)}}{r^{2 s-3}},
\end{aligned}
$$

where $\tau \in J=(0, T]$. Finally, by the triangle inequality and Lemma 3.2, and taking into account $\left\|E_{S}(0)\right\|_{0} \leqslant C\left\|S^{0}\right\|_{H^{s-1}} \frac{h^{\min (r, s-1)}}{r^{s-1}}$, we obtain the error estimates for the pressure and saturation:

$$
\begin{gathered}
\left\|E_{p}\right\|_{L^{2}\left(J ; L^{2}\right)}+\left\|\mathbf{K}^{1 / 2} \nabla E_{p}\right\|_{L^{2}\left(J ; L^{2}\right)} \leqslant C\left(\|p\|_{L^{2}\left(J ; H^{s}\right)}+\|S\|_{L^{2}\left(J ; H^{s}\right)}\right. \\
\left.+\left\|S_{t}\right\|_{L^{2}\left(J ; H^{s-1}\right)}\right) \frac{h^{\min (r, s-1)}}{r^{s-\frac{3}{2}}}, \\
\left\|E_{S}\right\|_{L^{\infty}\left(J ; L^{2}\right)}+\left\|\mathbf{K}^{1 / 2} \nabla E_{S}\right\|_{L^{2}\left(J ; L^{2}\right)} \leqslant C\left(\|p\|_{L^{2}\left(J ; H^{s}\right)}+\|S\|_{L^{2}\left(J ; H^{s}\right)}\right. \\
\left.+\left\|S_{t}\right\|_{L^{2}\left(J ; H^{s-1}\right)}+\left\|S^{0}\right\|_{H^{s-1}}\right) \frac{h^{\min (r, s-1)}}{r^{s-\frac{3}{2}}}
\end{gathered}
$$

where $r \geqslant 1$ and $s \geqslant 2$.

6. Existence of discrete solutions. For proving the global existence of approximate solutions, the key point is to construct a continuous mapping defined on a

Copyright $@$ ㅇ by SIAM. Unauthorized reproduction of this article is prohibited. 
bounded closed set. We first define two sets,

$$
\begin{aligned}
V_{h}^{p}:= & \left\{v(\cdot, t) \in \mathcal{D}_{r}\left(\mathcal{E}_{h}\right): \int_{0}^{T}\left\|\mathbf{K}^{1 / 2} \nabla(v-\widehat{p})\right\|_{0}^{2}\right. \\
& \left.+\int_{0}^{T} J_{0, D}^{\sigma}(v-\widehat{p}, v-\widehat{p}) \leqslant C \frac{h^{\min (2 r, 2 s-2)}}{r^{2 s-3}}\right\}, \\
V_{h}^{S}:= & \left\{v(\cdot, t) \in \mathcal{D}_{r}\left(\mathcal{E}_{h}\right):\|v-\widehat{S}\|_{L^{\infty}\left(J ; L^{2}\right)}^{2}+\int_{0}^{T}\left\|\mathbf{K}^{1 / 2} \nabla(v-\widehat{S})\right\|_{0}^{2}\right. \\
& \left.+\int_{0}^{T} J_{0, D}^{\sigma}(v-\widehat{S}, v-\widehat{S}) \leqslant C \frac{h^{\min (2 r, 2 s-2)}}{r^{2 s-3}}\right\},
\end{aligned}
$$

where $C$ depends on $\|p\|_{L^{2}\left(J ; H^{s}\right)},\|S\|_{L^{2}\left(J ; H^{s}\right)},\left\|S_{t}\right\|_{L^{2}\left(J ; H^{s-1}\right)}$, and $\left\|S^{0}\right\|_{H^{s-1}}$. It is clear that $V_{h}^{p}$ and $V_{h}^{S}$ are bounded and closed. Let $\bar{S}_{h}(\cdot, t) \in V_{h}^{S}$ be given and $\left(\bar{S}_{h}, \varphi\right)=\left(S^{0}, \varphi\right)$ at $t=0$ for any $\varphi \in \mathcal{D}_{r}\left(\mathcal{E}_{h}\right)$, then the pressure $p_{h}$ and saturation $S_{h}$ are computed by

$$
\begin{aligned}
& \left(\phi \frac{\partial S_{h}}{\partial t}, \varphi\right)-\sum_{E \in \mathcal{E}_{h}} \int_{E} \lambda_{c}\left(\bar{S}_{h}\right) \mathbf{K} \nabla S_{h} \cdot \nabla \varphi+\sum_{\gamma \in \Gamma_{h}} \int_{\gamma}\left\langle\lambda_{c}\left(\bar{S}_{h}\right) \mathbf{K} \nabla S_{h} \cdot \mathbf{n}_{\gamma}\right\rangle[\varphi] \\
& \quad+\sum_{\gamma \in \Gamma_{h}, D} \int_{\gamma}\left(\lambda_{c}\left(S_{B}\right) \mathbf{K} \nabla S_{h} \cdot \mathbf{n}_{\gamma}\right) \varphi+J_{0, D}^{\sigma}\left(S_{h}, \varphi\right)+B_{S}\left(\bar{S}_{h}, \varphi ; p_{h}\right)=L_{S}(\varphi) \\
& \quad+B_{p}\left(p_{h}, \varphi ; \bar{S}_{h}\right)-s_{\text {form }} \sum_{\gamma \in \Gamma_{h, D}} \int_{\gamma} \lambda_{t}\left(S_{B}\right) \mathbf{K} \nabla \varphi \cdot \mathbf{n}_{\gamma} p_{B}+J_{0}^{\sigma}\left(p_{c}\left(\bar{S}_{h}\right), \varphi\right)-J_{D}^{\sigma}\left(p_{B}, \varphi\right) \\
& \quad+s_{\text {form }} \sum_{\gamma \in \Gamma_{h}} \int_{\gamma}\left\langle\lambda_{n}\left(\bar{S}_{h}\right) \mathbf{K} \nabla \varphi \cdot \mathbf{n}_{\gamma}\right\rangle\left[p_{c}\left(\bar{S}_{h}\right)\right]-\int_{\Omega} q_{t} \varphi+J_{0, D}^{\sigma}\left(\bar{S}_{h}, \varphi\right), \quad t \in J
\end{aligned}
$$

for any $\varphi \in \mathcal{D}_{r}\left(\mathcal{E}_{h}\right)$.

The system of equations (6.1)-(6.3) is linear, and thus, from the theory of the linear DG system, it has a unique solution $\left(p_{h}, S_{h}\right)$. The approximate solution of pressure is unique for $\Gamma_{D} \neq \emptyset$ or for $\Gamma_{D}=\emptyset$ with the compatibility conditions. In order to establish the existence of numerical solutions, we introduce a map $\mathbb{T}_{h}$ such that $S_{h}=\mathbb{T}_{h}\left(\bar{S}_{h}\right)$, where $S_{h}$ solves the equations (6.1)-(6.3).

Using approaches similar to those used to analyze the error estimates in section 5 , we can prove that $p_{h} \in V_{h}^{p}$ and $S_{h} \in V_{h}^{S}$. For two given $\bar{S}_{h}^{i} \in V_{h}^{S}, \bar{S}_{h}^{j} \in V_{h}^{S}$, let $\left(p_{h}^{i}, S_{h}^{i}\right)$ and $\left(p_{h}^{j}, S_{h}^{j}\right)$ be the solutions of (6.1)-(6.3) when taking $\bar{S}_{h}=\bar{S}_{h}^{i}$ and $\bar{S}_{h}=\bar{S}_{h}^{j}$, respectively. Denote $S_{i j}=S_{h}^{i}-S_{h}^{j}, \quad \bar{S}_{i j}=\bar{S}_{h}^{i}-\bar{S}_{h}^{j}$, and $p_{i j}=p_{h}^{i}-p_{h}^{j}$.

Lemma 6.1. Let $s>1+\frac{d}{2}, 0<h \leqslant h_{0}, r \geqslant 2$. If the penalty parameter $\sigma_{0}$ is chosen to be sufficiently large, then we have

$$
\begin{aligned}
& \left\|S_{i j}\right\|_{L^{\infty}\left(J ; L^{2}\right)}^{2}+\int_{0}^{T}\left(\left\|\boldsymbol{K}^{1 / 2} \nabla p_{i j}\right\|_{0}^{2}+\left\|\boldsymbol{K}^{1 / 2} \nabla S_{i j}\right\|_{0}^{2}+J_{0, D}^{\sigma}\left(S_{i j}, S_{i j}\right)+J_{0, D}^{\sigma}\left(p_{i j}, p_{i j}\right)\right) \\
& \leqslant C\left(\left\|\bar{S}_{i j}\right\|_{L^{\infty}\left(J ; L^{2}\right)}^{2}+\left\|\boldsymbol{K}^{1 / 2} \nabla \bar{S}_{i j}\right\|_{0}^{2}+J_{0, D}^{\sigma}\left(\bar{S}_{i j}, \bar{S}_{i j}\right)\right),
\end{aligned}
$$

where $C$ depends on $r, p, S$.

Copyright $@$ by SIAM. Unauthorized reproduction of this article is prohibited. 
Lemma 6.1 can also be proved by approaches similar to those used in section 5 , and it demonstrates the continuity of the map $\mathbb{T}_{h}$. Therefore, we can conclude the global existence of discrete solutions in $V_{h}^{p}$ and $V_{h}^{S}$ by the Schauder fixed-point theorem.

7. The time discretization schemes. In this section, we will discuss the time discretization schemes applied to the nonlinear system obtained by the proposed DG schemes. To do this, we first divide the total time interval $[0, T]$ into $N$ uniform time steps as $0=t_{0}<t_{1}<\cdots<t_{N}=T$ and denote by $\tau=T / N$ the time step length, but our methods can be used for the cases of nonuniform time steps as well. Let $v^{i}$ denote the value of a function $v(t)$ at the time point $t_{i}$. For $t=0$, we can obtain $S_{h}^{0}$ by the initial conditions of the saturation. In what follows, we will propose two time discretization schemes for solving the system of nonlinear ordinary differential equations obtained by the proposed DG schemes.

7.1. IMPES-DG scheme. The IMplicit Pressure Explicit Saturation (IMPES) method [11] is a popular time-discretization scheme for simulating two-phase flow in porous media. Here, we use IMPES for (3.12) and (3.13) and obtain that

$$
\begin{aligned}
B_{p}\left(p_{h}^{i+1}, \varphi ; S_{h}^{i}\right) & =L_{p}\left(\varphi ; S_{h}^{i}\right), \\
\left(\phi \frac{S_{h}^{i+1}-S_{h}^{i}}{\tau}, \varphi\right)+B_{S}\left(S_{h}^{i}, \varphi ; p_{h}^{i+1}\right) & =L_{S}(\varphi)
\end{aligned}
$$

for $i \geqslant 0$ and any $\varphi \in \mathcal{D}_{r}\left(\mathcal{E}_{h}\right)$. In the saturation equation (7.2), the upwind values of saturations are determined by

$$
\left.S_{h}^{i *}\right|_{\gamma}=\left\{\begin{array}{lll}
\left.S_{h}^{i}\right|_{E_{\gamma}^{1}} & \text { if } & \mathbf{u}_{a}^{D G, i+1} \cdot \mathbf{n}_{\gamma} \geqslant 0 \\
\left.S_{h}^{i}\right|_{E_{\gamma}^{2}} & \text { if } & \mathbf{u}_{a}^{D G, i+1} \cdot \mathbf{n}_{\gamma}<0
\end{array}\right.
$$

where the two elements $E_{\gamma}^{1}$ and $E_{\gamma}^{2}$ share $\gamma$ with $\mathbf{n}_{\gamma}$ exterior to $E_{\gamma}^{1}$, and $\mathbf{u}_{a}^{D G, i+1} \cdot \mathbf{n}_{\gamma}$ is calculated by

$$
\mathbf{u}_{a}^{D G, i+1} \cdot \mathbf{n}_{\gamma}=-\left\langle\lambda_{t}\left(S_{h}^{i}\right) \mathbf{K} \nabla p_{h}^{i+1} \cdot \mathbf{n}\right\rangle+\frac{r^{2} \sigma_{\gamma}}{h_{\gamma}}\left[p_{h}^{i+1}\right] \quad \text { on } \gamma \in \Gamma_{h} .
$$

For given $S_{h}^{i}$, we can calculate $p_{h}^{i+1}$ by solving the pressure equation (7.1), which is a linear equation of $p_{h}^{i+1}$ and possesses a unique solution by the theory of linear DG methods for elliptic equations, and then we calculate $\mathbf{u}_{a}^{D G, i+1} \cdot \mathbf{n}_{\gamma}$ for each $\gamma \in$ $\Gamma_{h} \cup \Gamma_{h, D}$. Finally, we update the saturation $S_{h}^{i+1}$ explicitly by using the saturation equation (7.2). This process can start with $S_{h}^{0}$ given by (3.14) and work well if the suitable small time step size is chosen to guarantee the stability. It is also noted that at each time step, we always use the implicit scheme for the boundary conditions.

7.2. Improved IMPES-DG scheme. The IMPES-DG scheme treats the saturation explicitly in the pressure equation (7.1), and this treatment may result in instability of the IMPES method [22] and the decoupling between the pressure equation (3.12) and the saturation equation (3.13). In order to improve the IMPES-DG scheme, based on the ideas in [22], we treat the gradient of the saturation implicitly in (3.12), but keep $\lambda_{t}\left(S_{h}\right)$ and $\lambda_{c}\left(S_{h}\right)$ explicitly. As a result, we obtain the following 
pressure equation:

$$
\begin{aligned}
\sum_{E \in \mathcal{E}_{h}} & \int_{E} \lambda_{t}\left(S_{h}^{i}\right) \mathbf{K} \nabla p_{h}^{i+1} \cdot \nabla \varphi-\sum_{\gamma \in \Gamma_{h}} \int_{\gamma}\left\langle\lambda_{t}\left(S_{h}^{i}\right) \mathbf{K} \nabla p_{h}^{i+1} \cdot \mathbf{n}_{\gamma}\right\rangle[\varphi] \\
& -\sum_{\gamma \in \Gamma_{h}, D} \int_{\gamma}\left(\lambda_{t}\left(S_{B}^{i+1}\right) \mathbf{K} \nabla p_{h}^{i+1} \cdot \mathbf{n}_{\gamma}\right) \varphi \\
& +s_{\text {form }} \sum_{\gamma \in \Gamma_{h}} \int_{\gamma}\left\langle\lambda_{t}\left(S_{h}^{i}\right) \mathbf{K} \nabla \varphi \cdot \mathbf{n}_{\gamma}\right\rangle\left[p_{h}^{i+1}\right] \\
& +s_{\text {form }} \sum_{\gamma \in \Gamma_{h, D}} \int_{\gamma}\left(\lambda_{t}\left(S_{B}^{i+1}\right) \mathbf{K} \nabla \varphi \cdot \mathbf{n}_{\gamma}\right) p_{h}^{i+1}+J_{0, D}^{\sigma}\left(p_{h}^{i+1}, \varphi\right) \\
= & s_{\text {form }} \sum_{\gamma \in \Gamma_{h, D}} \int_{\gamma} \lambda_{t}\left(S_{B}^{i+1}\right) \mathbf{K} \nabla \varphi \cdot \mathbf{n}_{\gamma} p_{B}^{i+1}+J_{D}^{\sigma}\left(p_{B}^{i+1}, \varphi\right)+\int_{\Omega} q_{t}^{i+1} \varphi \\
& -\sum_{E \in \mathcal{E}_{h}} \int_{E} \lambda_{c}\left(S_{h}^{i}\right) \mathbf{K} \nabla S_{h}^{i+1} \cdot \nabla \varphi+\sum_{\gamma \in \Gamma_{h}} \int_{\gamma}\left\langle\lambda_{c}\left(S_{h}^{i}\right) \mathbf{K} \nabla S_{h}^{i+1} \cdot \mathbf{n}_{\gamma}\right\rangle[\varphi] \\
& +\sum_{\gamma \in \Gamma_{h}, D} \int_{\gamma}\left(\lambda_{c}\left(S_{B}^{i+1}\right) \mathbf{K} \nabla S_{h}^{i+1} \cdot \mathbf{n}_{\gamma}\right) \varphi \\
& -s_{\text {form }} \sum_{\gamma \in \Gamma_{h}} \int_{\gamma}\left\langle\lambda_{n}\left(S_{h}^{i}\right) \mathbf{K} \nabla \varphi \cdot \mathbf{n}_{\gamma}\right\rangle\left[D_{c}\left(S_{h}^{i+1}\right)\right] \\
& -J_{0}^{\sigma}\left(D_{c}\left(S_{h}^{i+1}\right), \varphi\right), \quad \varphi \in \mathcal{D}_{r}\left(\mathcal{E}_{h}\right),
\end{aligned}
$$

where $D_{c}\left(S_{h}^{i+1}\right)=p_{c}\left(S_{h}^{i}\right)+p_{c}^{\prime}\left(S_{h}^{i}\right)\left(S_{h}^{i+1}-S_{h}^{i}\right)$.

In the improved IMPES-DG scheme, for given $S_{h}^{i}$, the pressure $p_{h}^{i+1}$ and saturation $S_{h}^{i+1}$ solve the two linear equations (7.5) and (7.2). In order to efficiently solve this coupled system, we compute the upwind saturations in (7.2) as

$$
\left.S_{h}^{i *}\right|_{\gamma}=\left\{\begin{array}{lll}
\left.S_{h}^{i}\right|_{E_{\gamma}^{1}} & \text { if } & \mathbf{u}_{a}^{D G, i} \cdot \mathbf{n}_{\gamma} \geqslant 0 \\
\left.S_{h}^{i}\right|_{E_{\gamma}^{2}} & \text { if } & \mathbf{u}_{a}^{D G, i} \cdot \mathbf{n}_{\gamma}<0
\end{array}\right.
$$

For $i=0,\left.S_{h}^{0 *}\right|_{E_{\gamma}^{1}}=\left.S_{h}^{0}\right|_{E_{\gamma}^{1}}=\left.S_{h}^{0}\right|_{E_{\gamma}^{2}}$ since $\left[S^{0}\right]=0$ almost everywhere. In this case, we can substitute (7.2) into (7.5) to reduce the saturation $S_{h}^{i+1}$ of (7.5), and thus obtain a coupled pressure equation. Once the pressure $p_{h}^{i+1}$ is obtained, we can calculate $\mathbf{u}_{a}^{D G, i+1} \cdot \mathbf{n}_{\gamma}$ by (7.4) and update the saturation $S_{h}^{i+1}$ by (7.2), which are the same to the IMPES-DG scheme.

Finally, we give some remarks and further developments on the proposed IMPESDG scheme and improved IMPES-DG scheme as below.

(a) For the fully implicit time scheme applied to (3.12) and (3.13), we can construct the iterative methods based on the IMPES-DG scheme and improved IMPES-DG scheme, respectively, for solving the resulting nonlinear system. We refer to $[23,25]$ for similar iterative IMPES methods.

(b) The IMPES-DG scheme and improved IMPES-DG scheme allow us to use the multiscale time steps for pressures and saturations in different regions; see the detail in [9] for example.

(c) In future work, we will study the convergence of the IMPES-DG scheme and improved IMPES-DG scheme, especially the error estimates including the approximate time errors. 
8. Conclusions. We have developed a class of DG methods with interior penalties for incompressible two-phase flow in porous media with capillary pressures. The proposed methods incorporate the capillary pressures in the pressure equation instead of saturation equation. Differently from the conventional separate convergence analysis for the pressure and the saturation, we introduce a coupling error analysis approach, and based on this approach, we have obtained stability and a priori $h p$ error estimates of the schemes in $L^{2}\left(H^{1}\right)$ for the pressure and in $L^{\infty}\left(L^{2}\right)$ and $L^{2}\left(H^{1}\right)$ for the saturation. We also construct a continuous map to prove the global existence of the discrete solutions and present two time discretization schemes for effectively computing the discrete solutions.

Appendix A. Estimates of $\boldsymbol{G}_{\boldsymbol{p}}$. In Appendix A, we use $\left\{\epsilon_{i}^{p}\right\}_{i \geqslant 1}$ to indicate a sequence of small positive constants. The first term of $G_{p}$ is estimated as

$$
\begin{aligned}
\sum_{E \in \mathcal{E}_{h}} \int_{E} \lambda_{t}\left(S_{h}\right) \mathbf{K} \nabla E_{p}^{I} \cdot \nabla E_{p}^{A} & \leqslant \sum_{E \in \mathcal{E}_{h}}\left(\epsilon_{1}^{p}\left\|\mathbf{K}^{1 / 2} \nabla E_{p}^{A}\right\|_{0, E}^{2}+C\left\|\nabla E_{p}^{I}\right\|_{0, E}^{2}\right) \\
& \leqslant \epsilon_{1}^{p}\left\|\mathbf{K}^{1 / 2} \nabla E_{p}^{A}\right\|_{0}^{2}+C\|p\|_{s}^{2} \frac{h^{\min (2 r, 2 s-2)}}{r^{2 s-2}}
\end{aligned}
$$

where we have used the boundedness of $\lambda_{t}\left(S_{h}\right)$. The second term of $G_{p}$ is bounded as

$$
\begin{aligned}
& -\sum_{E \in \mathcal{E}_{h}} \int_{E}\left(\lambda_{t}(S)-\lambda_{t}\left(S_{h}\right)\right) \mathbf{K} \nabla p \cdot \nabla E_{p}^{A} \\
& \leqslant \sum_{E \in \mathcal{E}_{h}}\|\nabla p\|_{0, \infty, E}\left\|\mathbf{K}^{1 / 2} \nabla E_{p}^{A}\right\|_{0, E}\left\|\lambda_{t}(S)-\lambda_{t}\left(S_{h}\right)\right\|_{0, E} \\
& \leqslant C \sum_{E \in \mathcal{E}_{h}}\|\nabla p\|_{0, \infty, E}\left\|\mathbf{K}^{1 / 2} \nabla E_{p}^{A}\right\|_{0, E}\left\|E_{S}\right\|_{0, E} \\
& \leqslant \sum_{E \in \mathcal{E}_{h}}\left(\epsilon_{2}^{p}\left\|\mathbf{K}^{1 / 2} \nabla E_{p}^{A}\right\|_{0, E}+C\|\nabla p\|_{0, \infty, E}^{2}\left\|E_{S}\right\|_{0, E}^{2}\right) \\
& \leqslant \epsilon_{2}^{p}\left\|\mathbf{K}^{1 / 2} \nabla E_{p}^{A}\right\|_{0}^{2}+C\left\|E_{S}^{A}\right\|_{0}^{2}+C\|S\|_{s}^{2} \frac{h^{\min (2 r+2,2 s)}}{r^{2 s}}
\end{aligned}
$$

where we have used the assumption on the essential boundedness of $\nabla p$ and the first constant $C$ results from the Lipschitz continuity constant of $\lambda_{t}$. The third term of $G_{p}$ is estimated as

$$
\begin{aligned}
& \sum_{\gamma \in \Gamma_{h}} \int_{\gamma}\left\langle\left(\lambda_{t}(S)-\lambda_{t}\left(S_{h}\right)\right) \mathbf{K} \nabla p \cdot \mathbf{n}_{\gamma}\right\rangle\left[E_{p}^{A}\right] \\
& \quad \leqslant \frac{C}{\epsilon_{3}^{p}} \sum_{E \in \mathcal{E}_{h}} \frac{h_{E}}{\sigma_{0} r^{2}}\|\nabla p\|_{0, \infty, \partial E}^{2}\left\|\lambda_{t}(S)-\lambda_{t}\left(S_{h}\right)\right\|_{0, \partial E}^{2}+\epsilon_{3}^{p} \sum_{\gamma \in \Gamma_{h}} \frac{r^{2} \sigma_{\gamma}}{h_{\gamma}} \int_{\gamma}\left[E_{p}^{A}\right]^{2} \\
& \leqslant C \sum_{E \in \mathcal{E}_{h}} \frac{h_{E}}{r^{2}}\|\nabla p\|_{0, \infty, \partial E}^{2}\left\|E_{S}\right\|_{0, \partial E}^{2}+\epsilon_{3}^{p} J_{0, D}^{\sigma}\left(E_{p}^{A}, E_{p}^{A}\right) \\
& \leqslant C \sum_{E \in \mathcal{E}_{h}} \frac{h_{E}}{r^{2}}\left(\left\|E_{S}^{A}\right\|_{0, \partial E}^{2}+\left\|E_{S}^{I}\right\|_{0, \partial E}^{2}\right)+\epsilon_{3}^{p} J_{0, D}^{\sigma}\left(E_{p}^{A}, E_{p}^{A}\right) \\
& \leqslant C\left\|E_{S}^{A}\right\|_{0}^{2}+\epsilon_{3}^{p} J_{0, D}^{\sigma}\left(E_{p}^{A}, E_{p}^{A}\right)+C\|S\|_{s}^{2} \frac{h^{\min (2 r+2,2 s)}}{r^{2 s+1}}
\end{aligned}
$$

Copyright $@$ by SIAM. Unauthorized reproduction of this article is prohibited. 
where the upper boundedness of $\mathbf{K}$ and the assumption on the essential boundedness of $\nabla p$ have been applied, and here we choose the penalty parameters $\sigma_{\gamma} \geqslant \sigma_{0}>0$. Here, we have also used the inverse inequality and $h p$-approximation results. The fourth term consists of two pieces,

$$
\sum_{\gamma \in \Gamma_{h}} \int_{\gamma}\left\langle\lambda_{t}\left(S_{h}\right) \mathbf{K} \nabla E_{p} \cdot \mathbf{n}_{\gamma}\right\rangle\left[E_{p}^{A}\right]=\sum_{\gamma \in \Gamma_{h}} \int_{\gamma}\left\langle\lambda_{t}\left(S_{h}\right) \mathbf{K} \nabla\left(E_{p}^{A}-E_{p}^{I}\right) \cdot \mathbf{n}_{\gamma}\right\rangle\left[E_{p}^{A}\right] .
$$

Let the penalty parameter $\sigma_{0}$ be taken to be sufficiently large, then by the CauchySchwarz inequality and inverse inequality we have

$$
\begin{aligned}
& \sum_{\gamma \in \Gamma_{h}} \int_{\gamma}\left\langle\lambda_{t}\left(S_{h}\right) \mathbf{K} \nabla E_{p}^{A} \cdot \mathbf{n}_{\gamma}\right\rangle\left[E_{p}^{A}\right] \\
& \quad \leqslant C \sum_{E \in \mathcal{E}_{h}} \frac{h_{E}}{\sigma_{0} r^{2}}\left\|\mathbf{K}^{1 / 2} \nabla E_{p}^{A}\right\|_{0, \partial E}^{2}+\epsilon_{4}^{p} \sum_{\gamma \in \Gamma_{h}} \frac{r^{2} \sigma_{\gamma}}{h_{\gamma}} \int_{\gamma}\left[E_{p}^{A}\right]^{2} \\
& \quad \leqslant \sum_{E \in \mathcal{E}_{h}} \frac{C}{\sigma_{0}}\left\|\mathbf{K}^{1 / 2} \nabla E_{p}^{A}\right\|_{0, E}^{2}+\frac{1}{2} \epsilon_{4}^{p} J_{0, D}^{\sigma}\left(E_{p}^{A}, E_{p}^{A}\right) \\
& \quad \leqslant \epsilon_{5}^{p}\left\|\mathbf{K}^{1 / 2} \nabla E_{p}^{A}\right\|_{0}^{2}+\frac{1}{2} \epsilon_{4}^{p} J_{0, D}^{\sigma}\left(E_{p}^{A}, E_{p}^{A}\right) .
\end{aligned}
$$

Furthermore, the Cauchy-Schwarz inequality and approximation results yield that

$$
\begin{aligned}
\sum_{\gamma \in \Gamma_{h}} \int_{\gamma}\left\langle\lambda_{t}\left(S_{h}\right) \mathbf{K} \nabla E_{p}^{I} \cdot \mathbf{n}_{\gamma}\right\rangle\left[E_{p}^{A}\right] & \leqslant C \sum_{E \in \mathcal{E}_{h}} \frac{h_{E}}{r^{2}}\left\|\nabla E_{p}^{I}\right\|_{0, \partial E}^{2}+\frac{1}{2} \epsilon_{4}^{p} J_{0, D}^{\sigma}\left(E_{p}^{A}, E_{p}^{A}\right) \\
& \leqslant \frac{1}{2} \epsilon_{4}^{p} J_{0, D}^{\sigma}\left(E_{p}^{A}, E_{p}^{A}\right)+C\|p\|_{s}^{2} \frac{h^{\min (2 r, 2 s-2)}}{r^{2 s-1}}
\end{aligned}
$$

Therefore, the fourth term of $G_{p}$ can be estimated as

$$
\begin{gathered}
\sum_{\gamma \in \Gamma_{h}} \int_{\gamma}\left\langle\lambda_{t}\left(S_{h}\right) \mathbf{K} \nabla E_{p} \cdot \mathbf{n}_{\gamma}\right\rangle\left[E_{p}^{A}\right] \leqslant \\
\epsilon_{5}^{p}\left\|\mathbf{K}^{1 / 2} \nabla E_{p}^{A}\right\|_{0}^{2}+\epsilon_{4}^{p} J_{0, D}^{\sigma}\left(E_{p}^{A}, E_{p}^{A}\right) \\
+C\|p\|_{s}^{2} \frac{h^{\min (2 r, 2 s-2)}}{r^{2 s-1}} .
\end{gathered}
$$

For the fifth term of $G_{p}$, we have a similar result,

$$
\begin{gathered}
\sum_{\gamma \in \Gamma_{h, D}} \int_{\gamma}\left(\lambda_{t}\left(S_{B}\right) \mathbf{K} \nabla E_{p} \cdot \mathbf{n}_{\gamma}\right) E_{p}^{A} \leqslant \\
\epsilon_{6}^{p}\left\|\mathbf{K}^{1 / 2} \nabla E_{p}^{A}\right\|_{0}^{2}+\epsilon_{7}^{p} J_{0, D}^{\sigma}\left(E_{p}^{A}, E_{p}^{A}\right) \\
+C\|p\|_{s}^{2} \frac{h^{\min (2 r, 2 s-2)}}{r^{2 s-1}}
\end{gathered}
$$

In order to estimate the sixth term, by the Cauchy-Schwarz inequality, inverse inequality, and approximation results, we first get the estimate

$$
\begin{aligned}
\sum_{\gamma \in \Gamma_{h}} \int_{\gamma}\left\langle\lambda_{t}\left(S_{h}\right) \mathbf{K} \nabla E_{p}^{A} \cdot \mathbf{n}_{\gamma}\right\rangle\left[E_{p}^{I}\right] & \leqslant \sum_{E \in \mathcal{E}_{h}} \frac{h_{E}}{C r^{2}}\left\|\mathbf{K}^{1 / 2} \nabla E_{p}^{A}\right\|_{0, \partial E}^{2}+\sum_{E \in \mathcal{E}_{h}} \frac{C r^{2}}{h_{E}}\left\|E_{p}^{I}\right\|_{0, \partial E}^{2} \\
& \leqslant \epsilon_{8}^{p}\left\|\mathbf{K}^{1 / 2} \nabla E_{p}^{A}\right\|_{0}^{2}+C\|p\|_{s}^{2} \frac{h^{\min (2 r, 2 s-2)}}{r^{2 s-3}}
\end{aligned}
$$

Copyright $@$ by SIAM. Unauthorized reproduction of this article is prohibited. 
where we have used the boundedness of $\lambda_{t}$ again. Similarly to the fourth term, by using $E_{p}=E_{p}^{A}-E_{p}^{I}$, we obtain the estimate of the sixth term of $G_{p}$ as

$$
\begin{gathered}
-s_{\text {form }} \sum_{\gamma \in \Gamma_{h}} \int_{\gamma}\left\langle\lambda_{t}\left(S_{h}\right) \mathbf{K} \nabla E_{p}^{A} \cdot \mathbf{n}_{\gamma}\right\rangle\left[E_{p}\right] \leqslant \\
\left(\epsilon_{8}^{p}+\epsilon_{9}^{p}\right)\left\|\mathbf{K}^{1 / 2} \nabla E_{p}^{A}\right\|_{0}^{2}+\epsilon_{10}^{p} J_{0, D}^{\sigma}\left(E_{p}^{A}, E_{p}^{A}\right) \\
+C\|p\|_{s}^{2} \frac{h^{\min (2 r, 2 s-2)}}{r^{2 s-3}}
\end{gathered}
$$

and furthermore, we get a similar estimate for the seventh term,

$$
\begin{aligned}
-s_{\text {form }} \sum_{\gamma \in \Gamma_{h, D}} \int_{\gamma}\left(\lambda_{t}\left(S_{B}\right) \mathbf{K} \nabla E_{p}^{A} \cdot \mathbf{n}_{\gamma}\right) E_{p} \leqslant & \epsilon_{11}^{p}\left\|\mathbf{K}^{1 / 2} \nabla E_{p}^{A}\right\|_{0}^{2}+\epsilon_{12}^{p} J_{0, D}^{\sigma}\left(E_{p}^{A}, E_{p}^{A}\right) \\
& +C\|p\|_{s}^{2} \frac{h^{\min (2 r, 2 s-2)}}{r^{2 s-3}} .
\end{aligned}
$$

The eighth term is estimated by the Cauchy-Schwarz inequality and approximation results as

$$
J_{0, D}^{\sigma}\left(E_{p}^{I}, E_{p}^{A}\right) \leqslant \epsilon_{13}^{p} J_{0, D}^{\sigma}\left(E_{p}^{A}, E_{p}^{A}\right)+C\|p\|_{s}^{2} \frac{h^{\min (2 r, 2 s-2)}}{r^{2 s-3}} .
$$

For the ninth term, the Cauchy-Schwarz inequality and approximation results give us

$$
\sum_{E \in \mathcal{E}_{h}} \int_{E} \lambda_{c}\left(S_{h}\right) \mathbf{K} \nabla E_{S}^{I} \cdot \nabla E_{p}^{A} \leqslant \epsilon_{14}^{p}\left\|\mathbf{K}^{1 / 2} \nabla E_{p}^{A}\right\|_{0}^{2}+C\|S\|_{s}^{2} \frac{h^{\min (2 r, 2 s-2)}}{r^{2 s-2}},
$$

where we have used the boundedness of $\lambda_{c}$. Using the Lipschitz continuity of $\lambda_{c}$ and the essential boundedness of $\nabla S$ on each element, we bound the tenth term as

$$
\begin{aligned}
& -\sum_{E \in \mathcal{E}_{h}} \int_{E}\left(\lambda_{c}(S)-\lambda_{c}\left(S_{h}\right)\right) \mathbf{K} \nabla S \cdot \nabla E_{p}^{A} \\
& \quad \leqslant C \sum_{E \in \mathcal{E}_{h}}\|\nabla S\|_{0, \infty, E}\left\|\mathbf{K}^{1 / 2} \nabla E_{p}^{A}\right\|_{0, E}\left\|E_{S}\right\|_{0, E} \\
& \quad \leqslant \epsilon_{15}^{p}\left\|\mathbf{K}^{1 / 2} \nabla E_{p}^{A}\right\|_{0}^{2}+C\left\|E_{S}^{A}\right\|_{0}^{2}+C\|S\|_{s}^{2} \frac{h^{\min (2 r+2,2 s)}}{r^{2 s}} .
\end{aligned}
$$

Since $\lambda_{c}$ and $\mathbf{K}$ are bounded, it follows by the Cauchy-Schwarz inequality, inverse inequality, and approximation results that

$$
\begin{aligned}
& \sum_{\gamma \in \Gamma_{h}} \int_{\gamma}\left\langle\lambda_{c}\left(S_{h}\right) \mathbf{K} \nabla E_{S} \cdot \mathbf{n}_{\gamma}\right\rangle\left[E_{p}^{A}\right] \\
& \quad \leqslant \frac{C}{\sigma_{0}} \sum_{E \in \mathcal{E}_{h}} \frac{h_{E}}{r^{2}}\left\|\nabla E_{S}\right\|_{0, \partial E}^{2}+\epsilon_{16}^{p} J_{0, D}^{\sigma}\left(E_{p}^{A}, E_{p}^{A}\right) \\
& \quad \leqslant \epsilon_{16}^{p} J_{0, D}^{\sigma}\left(E_{p}^{A}, E_{p}^{A}\right)+\epsilon_{17}^{p}\left\|\mathbf{K}^{1 / 2} \nabla E_{S}^{A}\right\|_{0}^{2}+C\|S\|_{s}^{2} \frac{h^{\min (2 r, 2 s-2)}}{r^{2 s-1}},
\end{aligned}
$$

where we choose the penalty parameters $\sigma_{0}$ to be sufficiently large. Applying tech- 
niques similar to those used in the twelfth term, we get

$$
\begin{aligned}
\sum_{\gamma \in \Gamma_{h, D}} \int_{\gamma} \lambda_{c}\left(S_{B}\right) \mathbf{K} \nabla E_{S} \cdot \mathbf{n}_{\gamma} E_{p}^{A} \leqslant & \epsilon_{18}^{p} J_{0, D}^{\sigma}\left(E_{p}^{A}, E_{p}^{A}\right)+\epsilon_{19}^{p}\left\|\mathbf{K}^{1 / 2} \nabla E_{S}^{A}\right\|_{0}^{2} \\
& +C\|S\|_{s}^{2} \frac{h^{\min (2 r, 2 s-2)}}{r^{2 s-1}} .
\end{aligned}
$$

We use the Lipschitz continuity of $\lambda_{c}$ and the the boundedness of $\nabla S$ and $\mathbf{K}$ again to estimate the thirteen term:

$$
\begin{aligned}
& \sum_{\gamma \in \Gamma_{h}} \int_{\gamma}\left\langle\left(\lambda_{c}(S)-\lambda_{c}\left(S_{h}\right)\right) \mathbf{K} \nabla S \cdot \mathbf{n}_{\gamma}\right\rangle\left[E_{p}^{A}\right] \\
& \leqslant C \sum_{E \in \mathcal{E}_{h}} \frac{h_{E}}{r^{2}}\|\nabla S\|_{0, \infty, \partial E}^{2}\left\|E_{S}\right\|_{0, \partial E}^{2}+\epsilon_{20}^{p} J_{0, D}^{\sigma}\left(E_{p}^{A}, E_{p}^{A}\right) \\
& \leqslant \epsilon_{20}^{p} J_{0, D}^{\sigma}\left(E_{p}^{A}, E_{p}^{A}\right)+C\left\|E_{S}^{A}\right\|_{0}^{2}+C\|S\|_{s}^{2} \frac{h^{\min (2 r+2,2 s)}}{r^{2 s+1}}
\end{aligned}
$$

where we have also used the approximation results and inverse inequality. The last term is estimated as

$$
-J_{0}^{\sigma}\left(p_{c}(S)-p_{c}\left(S_{h}\right), E_{p}^{A}\right) \leqslant a_{1} J_{0}^{\sigma}\left(E_{S}^{A}, E_{S}^{A}\right)+\frac{1}{2} J_{0}^{\sigma}\left(E_{p}^{A}, E_{p}^{A}\right)+C\|S\|_{s}^{2} \frac{h^{\min (2 r, 2 s-2)}}{r^{2 s-3}} .
$$

Summing the above estimates of all terms of $G_{p}$, we can see that

$$
\begin{aligned}
G_{p} \leqslant & \left(\epsilon_{1}^{p}+\epsilon_{2}^{p}+\epsilon_{5}^{p}+\epsilon_{6}^{p}+\epsilon_{8}^{p}+\epsilon_{9}^{p}+\epsilon_{11}^{p}+\epsilon_{14}^{p}+\epsilon_{15}^{p}\right)\left\|\mathbf{K}^{1 / 2} \nabla E_{p}^{A}\right\|_{0}^{2} \\
& +\left(\frac{1}{2}+\epsilon_{3}^{p}+\epsilon_{4}^{p}+\epsilon_{7}^{p}+\epsilon_{10}^{p}+\epsilon_{12}^{p}+\epsilon_{13}^{p}+\epsilon_{16}^{p}+\epsilon_{18}^{p}+\epsilon_{20}^{p}\right) J_{0, D}^{\sigma}\left(E_{p}^{A}, E_{p}^{A}\right) \\
& +\left(\epsilon_{17}^{p}+\epsilon_{19}^{p}\right)\left\|\mathbf{K}^{1 / 2} \nabla E_{S}^{A}\right\|_{0}^{2}+C\left\|E_{S}^{A}\right\|_{0}^{2}+a_{1} J_{0}^{\sigma}\left(E_{S}^{A}, E_{S}^{A}\right) \\
& +C\left(\|p\|_{s}^{2}+\|S\|_{s}^{2}\right) \frac{h^{\min (2 r, 2 s-2)}}{r^{2 s-3}} .
\end{aligned}
$$

Appendix B. Estimates of $\boldsymbol{G}_{\boldsymbol{S}}$. We now estimate the terms of $G_{S}$, and in Appendix B, let $\left\{\epsilon_{i}^{s}\right\}_{i \geqslant 1}$ denote a sequence of small positive constants. Using the Cauchy-Schwarz inequality and approximation results, we can obtain the estimate of the first term as

$$
-\sum_{E \in \mathcal{E}_{h}} \int_{E} \lambda_{n}\left(S_{h}\right) \mathbf{K} \nabla E_{p}^{I} \cdot \nabla E_{S}^{A} \leqslant \epsilon_{1}^{s}\left\|\mathbf{K}^{1 / 2} \nabla E_{S}^{A}\right\|_{0}^{2}+C\|p\|_{s}^{2} \frac{h^{\min (2 r, 2 s-2)}}{r^{2 s-2}},
$$

where the boundedness of $\lambda_{n}$ has been used. Using the Lipschitz continuity of $\lambda_{n}(S)$ and approximation results, we estimate the second term as

$$
\begin{aligned}
& \sum_{E \in \mathcal{E}_{h}} \int_{E}\left(\lambda_{n}(S)-\lambda_{n}\left(S_{h}\right)\right) \mathbf{K} \nabla p \cdot \nabla E_{S}^{A} \\
& \quad \leqslant C \sum_{E \in \mathcal{E}_{h}}\|\nabla p\|_{0, \infty, E}\left\|E_{S}\right\|_{0, E}\left\|\mathbf{K}^{1 / 2} \nabla E_{S}^{A}\right\|_{0, E} \\
& \quad \leqslant C \sum_{E \in \mathcal{E}_{h}}\left\|E_{S}\right\|_{0, E}^{2}+\epsilon_{2}^{s} \sum_{E \in \mathcal{E}_{h}}\left\|\mathbf{K}^{1 / 2} \nabla E_{S}^{A}\right\|_{0, E}^{2} \\
& \quad \leqslant C\left\|E_{S}^{A}\right\|_{0}^{2}+\epsilon_{2}^{s}\left\|\mathbf{K}^{1 / 2} \nabla E_{S}^{A}\right\|_{0}^{2}+C\|S\|_{s}^{2} \frac{h^{\min (2 r+2,2 s)}}{r^{2 s}}
\end{aligned}
$$

Copyright $@$ by SIAM. Unauthorized reproduction of this article is prohibited. 
where we have also used the boundedness of the absolute permeability $\mathbf{K}$ and $\nabla p$. Applying the inverse inequality and approximation results, we estimate the third term to be

$$
\begin{aligned}
& -\sum_{\gamma \in \Gamma_{h}} \int_{\gamma}\left(f_{n}\left(S^{*}\right)\left\langle\lambda_{t}(S) \mathbf{K} \nabla p \cdot \mathbf{n}_{\gamma}\right\rangle-f_{n}\left(S_{h}^{*}\right)\left\langle\lambda_{t}\left(S_{h}\right) \mathbf{K} \nabla p \cdot \mathbf{n}_{\gamma}\right\rangle\right)\left[E_{S}^{A}\right] \\
& \leqslant C \sum_{E \in \mathcal{E}_{h}} \frac{h_{E}}{r^{2}}\|\nabla p\|_{0, \infty, \partial E}^{2}\left\|E_{S}\right\|_{0, \partial E}^{2}+\epsilon_{3}^{s} \sum_{\gamma \in \Gamma_{h}} \frac{r^{2} \sigma_{\gamma}}{h_{\gamma}} \int_{\gamma}\left[E_{S}^{A}\right]^{2} \\
& \quad \leqslant C\left\|E_{S}^{A}\right\|_{0}^{2}+\epsilon_{3}^{s} J_{0, D}^{\sigma}\left(E_{S}^{A}, E_{S}^{A}\right)+C\|S\|_{s}^{2} \frac{h^{\min (2 r+2,2 s)}}{r^{2 s+1}}
\end{aligned}
$$

where the Lipschitz continuity of $\lambda_{\alpha}$ and $f_{n}$ has also been used. By the virtue of the boundedness of $\lambda_{t}$ and $f_{n}$, the inverse inequality, and approximation results, the fourth term is estimated as

$$
\begin{aligned}
& -\sum_{\gamma \in \Gamma_{h}} \int_{\gamma} f_{n}\left(S_{h}^{*}\right)\left\langle\lambda_{t}\left(S_{h}\right) \mathbf{K} \nabla E_{p} \cdot \mathbf{n}_{\gamma}\right\rangle\left[E_{S}^{A}\right] \\
& \leqslant \frac{C}{\sigma_{0}} \sum_{E \in \mathcal{E}_{h}} \frac{h_{E}}{r^{2}}\left(\left\|\mathbf{K}^{1 / 2} E_{p}^{A}\right\|_{0, \partial E}^{2}+\left\|\nabla E_{p}^{I}\right\|_{0, \partial E}^{2}\right)+\epsilon_{4}^{s} \sum_{\gamma \in \Gamma_{h}} \frac{r^{2} \sigma_{\gamma}}{h_{\gamma}} \int_{\gamma}\left[E_{S}^{A}\right]^{2} \\
& \quad \leqslant \epsilon_{5}^{s}\left\|\mathbf{K}^{1 / 2} E_{p}^{A}\right\|_{0}^{2}+\epsilon_{4}^{s} J_{0, D}^{\sigma}\left(E_{S}^{A}, E_{S}^{A}\right)+C\|p\|_{s}^{2} \frac{h^{\min (2 r, 2 s-2)}}{r^{2 s-1}},
\end{aligned}
$$

where we choose the penalty parameters $\sigma_{0}$ to be sufficiently large for the sake of getting a suitable small $\epsilon_{5}^{s}$ for $\left\|\mathbf{K}^{1 / 2} E_{p}^{A}\right\|_{0}^{2}$. Similarly, for the fifth term, we have

$$
\begin{aligned}
-\sum_{\gamma \in \Gamma_{h, D}} \int_{\gamma}\left(\lambda_{n}\left(S_{B}\right) \mathbf{K} \nabla E_{p} \cdot \mathbf{n}_{\gamma}\right) E_{S}^{A} \leqslant & \epsilon_{6}^{s}\left\|\mathbf{K}^{1 / 2} E_{p}^{A}\right\|_{0}^{2}+\epsilon_{7}^{s} J_{0, D}^{\sigma}\left(E_{S}^{A}, E_{S}^{A}\right) \\
& +C\|p\|_{s}^{2} \frac{h^{\min (2 r, 2 s-2)}}{r^{2 s-1}} .
\end{aligned}
$$

For the sixth term, we get

$$
\begin{aligned}
& s_{\text {form }} \sum_{\gamma \in \Gamma_{h}} \int_{\gamma} f_{n}\left(S_{h}^{*}\right)\left\langle\lambda_{t}\left(S_{h}\right) \mathbf{K} \nabla E_{S}^{A} \cdot \mathbf{n}_{\gamma}\right\rangle\left[E_{p}\right] \\
& \leqslant \frac{C}{\sigma_{0}} \sum_{E \in \mathcal{E}_{h}} \frac{h_{E}}{r^{2}}\left\|\mathbf{K}^{1 / 2} \nabla E_{S}^{A}\right\|_{0, \partial E}^{2}+\epsilon_{8}^{s} J_{0, D}^{\sigma}\left(E_{p}^{A}, E_{p}^{A}\right)+C \sum_{\gamma \in \Gamma_{h}} \frac{r^{2} \sigma_{\gamma}}{h_{\gamma}} \int_{\gamma}\left[E_{p}^{I}\right]^{2} \\
& \leqslant \epsilon_{9}^{s}\left\|\mathbf{K}^{1 / 2} \nabla E_{S}^{A}\right\|_{0}^{2}+\epsilon_{8}^{s} J_{0, D}^{\sigma}\left(E_{p}^{A}, E_{p}^{A}\right)+C \sum_{E \in \mathcal{E}_{h}} \frac{r^{2}}{h_{E}}\left\|E_{p}^{I}\right\|_{0, \partial E}^{2} \\
& \leqslant \epsilon_{9}^{s}\left\|\mathbf{K}^{1 / 2} \nabla E_{S}^{A}\right\|_{0}^{2}+\epsilon_{8}^{s} J_{0, D}^{\sigma}\left(E_{p}^{A}, E_{p}^{A}\right)+C\|p\|_{s}^{2} \frac{h^{\min (2 r, 2 s-2)}}{r^{2 s-3}} .
\end{aligned}
$$

A similar estimate can be obtained for the seventh term,

$$
\begin{gathered}
s_{\text {form }} \sum_{\gamma \in \Gamma_{h, D}} \int_{\gamma}\left(\lambda_{n}\left(S_{B}\right) \mathbf{K} \nabla E_{S}^{A} \cdot \mathbf{n}_{\gamma}\right) E_{p} \leqslant \\
\epsilon_{10}^{s}\left\|\mathbf{K}^{1 / 2} \nabla E_{S}^{A}\right\|_{0}^{2}+\epsilon_{11}^{s} J_{0, D}^{\sigma}\left(E_{p}^{A}, E_{p}^{A}\right) \\
+C\|p\|_{s}^{2} \frac{h^{\min (2 r, 2 s-2)}}{r^{2 s-3}} .
\end{gathered}
$$

The terms from eight to thirteen of $G_{S}$ can be estimated by similar techniques. Here we need to use the Lipschitz continuity of $\lambda_{c}(S)$ and the boundedness of $\lambda_{c}$ and $\nabla S$. 
For the sake of simplicity, we drop the derivations and only list the estimate results:

$$
\begin{aligned}
& -\sum_{E \in \mathcal{E}_{h}} \int_{E} \lambda_{c}\left(S_{h}\right) \mathbf{K} \nabla E_{S}^{I} \cdot \nabla E_{S}^{A} \\
& \quad \leqslant \epsilon_{12}^{s}\left\|\mathbf{K}^{1 / 2} \nabla E_{S}^{A}\right\|_{0}^{2}+C\|S\|_{s}^{2} \frac{h^{\min (2 r, 2 s-2)}}{r^{2 s-2}}, \\
& \sum_{E \in \mathcal{E}_{h}} \int_{E}\left(\lambda_{c}(S)-\lambda_{c}\left(S_{h}\right)\right) \mathbf{K} \nabla p \cdot \nabla E_{S}^{A} \\
& \quad \leqslant C\left\|E_{S}^{A}\right\|_{0}^{2}+\epsilon_{13}^{s}\left\|\mathbf{K}^{1 / 2} \nabla E_{S}^{A}\right\|_{0}^{2}+C\|S\|_{s}^{2} \frac{h^{\min (2 r+2,2 s)}}{r^{2 s}}, \\
& -\sum_{\gamma \in \Gamma_{h}} \int_{\gamma}\left\langle\lambda_{c}\left(S_{h}\right) \mathbf{K} \nabla E_{S} \cdot \mathbf{n}_{\gamma}\right\rangle\left[E_{S}^{A}\right] \\
& \quad \leqslant \epsilon_{14}^{s}\left\|\mathbf{K}^{1 / 2} \nabla E_{S}^{A}\right\|_{0}^{2}+\epsilon_{15}^{s} J_{0, D}^{\sigma}\left(E_{S}^{A}, E_{S}^{A}\right)+C\|S\|_{s}^{2} \frac{h^{\min (2 r, 2 s-2)}}{r^{2 s-1}}, \\
& -\sum_{\gamma \in \Gamma_{h}} \int_{\gamma}\left\langle\left(\lambda_{c}(S)-\lambda_{c}\left(S_{h}\right)\right) \mathbf{K} \nabla S \cdot \mathbf{n}_{\gamma}\right\rangle\left[E_{S}^{A}\right] \\
& \quad \leqslant C\left\|E_{S}^{A}\right\|_{0}^{2}+\epsilon_{16}^{s} J_{0, D}^{\sigma}\left(E_{S}^{A}, E_{S}^{A}\right)+C\|S\|_{s}^{2} \frac{h^{\min (2 r+2,2 s)}}{r^{2 s+1}}, \\
& -\sum_{\gamma \in \Gamma_{h, D}} \int_{\gamma}\left(\lambda_{c}\left(S_{B}\right) \mathbf{K} \nabla E_{S} \cdot \mathbf{n}_{\gamma}\right) E_{S}^{A} \\
& \quad \leqslant \epsilon_{17}^{s}\left\|\mathbf{K}^{1 / 2} \nabla E_{S}^{A}\right\|_{0}^{2}+\epsilon_{18}^{s} J_{0, D}^{\sigma}\left(E_{S}^{A}, E_{S}^{A}\right)+C\|S\|_{s}^{2} \frac{h^{\min (2 r, 2 s-2)}}{r^{2 s-1}}, \\
& -s_{\text {form }} \sum_{\gamma \in \Gamma_{h}} \int_{\gamma}\left\langle\lambda_{n}\left(S_{h}\right) \mathbf{K} \nabla\left(E_{p}^{A}-E_{S}^{A}\right) \cdot \mathbf{n}_{\gamma}\right\rangle\left[p_{c}(S)-p_{c}\left(S_{h}\right)\right] \\
& \quad \leqslant \epsilon_{19}^{s}\left\|\mathbf{K}^{1 / 2} \nabla E_{p}^{A}\right\|_{0}^{2}+\epsilon_{20}^{s}\left\|\mathbf{K}^{1 / 2} \nabla E_{S}^{A}\right\|_{0}^{2}+\epsilon_{21}^{s} J_{0, D}^{\sigma}\left(E_{S}^{A}, E_{S}^{A}\right)+C\|S\|_{s}^{2} \frac{h^{\min (2 r, 2 s-2)}}{r^{2 s-3}} .
\end{aligned}
$$

The rest of the terms are trivially estimated by

$$
\begin{aligned}
(\omega+1) J_{0}^{\sigma}\left(p_{c}(S)-p_{c}(\widehat{S}), E_{S}^{A}\right)+J_{D}^{\sigma}\left(E_{S}^{I}, E_{S}^{A}\right) & \\
& \leqslant \epsilon_{22}^{s} J_{0, D}^{\sigma}\left(E_{S}^{A}, E_{S}^{A}\right)+C\|S\|_{s}^{2} \frac{h^{\min (2 r, 2 s-2)}}{r^{2 s-3}} .
\end{aligned}
$$

Taking into account the boundedness of porosity, and by the Cauchy-Schwarz inequality and approximation results, we can estimate the last term of $G_{S}$ as

$$
\left(\phi \frac{\partial E_{S}^{I}}{\partial t}, E_{S}^{A}\right) \leqslant C\left\|E_{S}^{A}\right\|_{0}^{2}+C\left\|\frac{\partial E_{S}^{I}}{\partial t}\right\|_{0}^{2} \leqslant C\left\|E_{S}^{A}\right\|_{0}^{2}+C\left\|S_{t}\right\|_{s-1}^{2} \frac{h^{\min (2 r+2,2 s-2)}}{r^{2 s-2}} .
$$

Finally, we sum the above estimates of $G_{S}$ and obtain that

$$
\begin{aligned}
G_{S} \leqslant & \left(\epsilon_{1}^{s}+\epsilon_{2}^{s}+\epsilon_{9}^{s}+\epsilon_{10}^{s}+\epsilon_{12}^{s}+\epsilon_{13}^{s}+\epsilon_{14}^{s}+\epsilon_{17}^{s}+\epsilon_{20}^{s}\right)\left\|\mathbf{K}^{1 / 2} \nabla E_{S}^{A}\right\|_{0}^{2} \\
& +\left(\epsilon_{3}^{s}+\epsilon_{4}^{s}+\epsilon_{7}^{s}+\epsilon_{15}^{s}+\epsilon_{16}^{s}+\epsilon_{18}^{s}+\epsilon_{21}^{s}+\epsilon_{22}^{s}\right) J_{0, D}^{\sigma}\left(E_{S}^{A}, E_{S}^{A}\right) \\
& +\left(\epsilon_{5}^{s}+\epsilon_{6}^{s}+\epsilon_{19}^{s}\right)\left\|\mathbf{K}^{1 / 2} \nabla E_{p}^{A}\right\|_{0}^{2}+\left(\epsilon_{8}^{s}+\epsilon_{11}^{s}\right) J_{0, D}^{\sigma}\left(E_{p}^{A}, E_{p}^{A}\right) \\
& +C\left\|E_{S}^{A}\right\|_{0}^{2}+C\left(\|p\|_{s}^{2}+\|S\|_{s}^{2}+\left\|S_{t}\right\|_{s-1}^{2}\right) \frac{h^{\min (2 r, 2 s-2)}}{r^{2 s-3}}
\end{aligned}
$$

Copyright $@$ by SIAM. Unauthorized reproduction of this article is prohibited. 
Acknowledgment. The authors would like to thank the referees for their constructive suggestions and comments to improve the original version of this paper. The authors also cheerfully appreciate the generous support of the university research fund to the Computational Transport Phenomena Laboratory at KAUST.

\section{REFERENCES}

[1] T. Arbogast, Implementation of a locally conservative numerical subgrid upscaling scheme for two-phase Darcy flow, Comput. Geosci., 6 (2002), pp. 453-481.

[2] I. Babuška, C.E. Baumann, And J.T. Oden, A discontinuous hp finite element method for diffusion problems: 1-d analysis, Comput. Math. Appl., 37 (1999), pp. 103-122.

[3] I. BABUŠKA AND M. SURI, The $h$-p version of the finite element method with quasi-uniform meshes, ESAIM Math. Model. Numer. Anal., 21 (1987), pp. 199-238.

[4] I. BABUŠKA AND M. SURI, The optimal convergence rates of the p-version of the finite element method, SIAM J. Numer. Anal., 24 (1987), pp. 750-776.

[5] S. Bartels, M. Jensen, And R. Müller, Discontinuous Galerkin finite element convergence for incompressible miscible displacement problems of low regularity, SIAM J. Numer. Anal., 47 (2009), pp. 3720-3743.

[6] S.C. Brenner, Poincaré-Friedrichs inequalities for piecewise $H^{1}$ functions, SIAM J. Numer. Anal., 41 (2003), pp. 306-324.

[7] G. Chavent, G. Cohen, J. Jaffre, R. Eyard, D. Guerillot, and L. Weill, Discontinuous and mixed finite elements for two-phase incompressible flow, SPE Reservoir Engrg., 5 (1990), pp. 567-575.

[8] Z. Chen AND R.E. EwIng, Degenerate two-phase incompressible flow iii. Sharp error estimates, Numer. Math., 90 (2001), pp. 215-240.

[9] Z. Chen, G. Huan, And Y. MA, Computational Methods for Multiphase Flows in Porous Media, SIAM Comp. Sci. Eng., SIAM, Philadelphia, 2006.

[10] P.G. Ciarlet, The Finite Element Method for Elliptic Problems, Vol. 4, North-Holland, Amsterdam, 1978.

[11] K. COATs, A note on IMPES and some IMPES-based simulation models, SPE J., 5 (2000), pp. $245-251$.

[12] M. CuI, A combined mixed and discontinuous Galerkin method for compressible miscible displacement problem in porous media, J. Comput. Appl. Math., 198 (2007), pp. 19-34.

[13] C.N. Dawson, H. Klíe, M.F. Wheeler, And C.S. Woodward, A parallel, implicit, cellcentered method for two-phase flow with a preconditioned Newton-Krylov solver, Comput. Geosci., 1 (1997), pp. 215-249.

[14] C. Dawson, S. Sun, And M.F. Wheeler, Compatible algorithms for coupled flow and transport, Comput. Methods Appl. Mech. Engrg., 193 (2004), pp. 2565-2580.

[15] H. EGger AND J. SchöBerL, A hybrid mixed discontinuous Galerkin finite-element method for convection-diffusion problems, IMA J. Numer. Anal., 30 (2010), pp. 1206-1234.

[16] Y. EPSHTEYN AND B. Rivière, Fully implicit discontinuous finite element methods for twophase flow, Appl. Numer. Math., 57 (2007), pp. 383-401.

[17] Y. Epshteyn And B. Rivière, Analysis of hp discontinuous Galerkin methods for incompressible two-phase flow, J. Comput. Appl. Math., 225 (2009), pp. 487-509.

[18] A. ERn, I. Mozolevski, AND L. SchuH, Discontinuous Galerkin approximation of two-phase flows in heterogeneous porous media with discontinuous capillary pressures, Comput. Methods Appl. Mech. Engrg., 199 (2010), pp. 1491-1501.

[19] R. FuČÍK AND J. MIKYŠKA, Discontinous Galerkin and mixed-hybrid finite element approach to two-phase flow in heterogeneous porous media with different capillary, Procedia Comput. Sci., 4 (2011), pp. 908-917.

[20] R. Holm, R. Kaufmann, E.I. Dale, S. Aanonsen, G.E. Fladmark, M. Espedal, and A. Skauge, Constructing three-phase capillary pressure functions by parameter matching using a modified ensemble Kalman filter, Commun. Comput. Phys., 6 (2009), pp. 24-48.

[21] H. Hoteit And A. Firoozabadi, Numerical modeling of two-phase flow in heterogeneous permeable media with different capillarity pressures, Adv. Water Resources, 31 (2008), pp. 5673.

[22] J. Kou And S. Sun, A new treatment of capillarity to improve the stability of IMPES two-phase flow formulation, Comput. \& Fluids, 39 (2010), pp. 1293-1931.

[23] J. Kou AND S. Sun, On iterative IMPES formulation for two phase flow with capillarity in heterogeneous porous media, Internat. J. Numer. Anal. Model. Ser. B, 1 (2010), pp. 20-40.

Copyright (C) by SIAM. Unauthorized reproduction of this article is prohibited. 
[24] J. Kou And S. Sun, Analysis of a combined mixed finite element and discontinuous Galerkin method for incompressible two-phase flow in porous media, Math. Methods Appl. Sci., to appear.

[25] S. Lacroix, Yu. Vassilevski, J. Wheeler, and M. Wheeler, Iterative solution methods for modeling multiphase flow in porous media fully implicitly, SIAM J. Sci. Comput., 25 (2003), pp. 905-926.

[26] D. Nayagum, G. Schäfer, And R. Mosé, Modelling two-phase incompressible flow in porous media using mixed hybrid and discontinuous finite elements, Comput. Geosci., 8 (2004), pp. $49-73$.

[27] J. Niessner, R. Helmig, H. Jakobs, and J.E. Roberts, Interface condition and linearization schemes in the Newton iterations for two-phase flow in heterogeneous porous media, Adv. Water Resources, 28 (2005), pp. 671-687.

[28] J.T. Oden, I. BabušKa, and C.E. Baumann, A discontinuous hp finite element method for diffusion problems, J. Comput. Phys., 146 (1998), pp. 491-516.

[29] V. Reichenberger, H. Jakobs, P. Bastian, and R. Helmig, A mixed-dimensional finite volume method for two-phase flow in fractured porous media, Adv. Water Resources, 29 (2006), pp. 1020-1036.

[30] B.M. RiviÈRe and N.J. WALkington, Convergence of a discontinuous Galerkin method for the miscible displacement equation under low regularity, SIAM J. Numer. Anal., 49 (2011), pp. $1085-1110$.

[31] B. Rivière, M.F. Wheeler, and V. Girault, A priori error estimates for finite element methods based on discontinuous approximation spaces for elliptic problems, SIAM J. Numer. Anal., 39 (2001), pp. 902-931.

[32] P. Sun, G. XUe, C. WANG, AND J. XU, Fast numerical simulation of two-phase transport model in the cathode of a polymer electrolyte fuel cell, Commun. Comput. Phys., 6 (2009), pp. $49-71$.

[33] S. Sun, A. Firoozabadi, And J. Kou, Numerical modeling of two-phase binary fluid mixing using mixed finite elements, Comput. Geosci., 16 (2012), pp. 1101-1124.

[34] S. Sun And J. Liu, A locally conservative finite element method based on piecewise constant enrichment of the continuous Galerkin method, SIAM J. Sci. Comput., 31 (2009), pp. 25282548 .

[35] S. Sun AND M.F. Wheeler, Anisotropic and dynamic mesh adaptation for discontinuous Galerkin methods applied to reactive transport, Comput. Methods Appl. Mech. Engrg., 195 (2006), pp. 3382-3405.

[36] S. Sun AND M.F. WheEler, Symmetric and nonsymmetric discontinuous Galerkin methods for reactive transport in porous media, SIAM J. Numer. Anal., 43 (2006), pp. 195-219.

[37] S. SUn AND M.F. WHEELER, A dynamic, adaptive, locally conservative, and nonconforming solution strategy for transport phenomena in chemical engineering, Chem. Engrg. Commun., 193 (2006), pp. 1527-1545.

[38] S. Sun And M.F. WheEler, Discontinuous Galerkin methods for simulating bioreactive transport of viruses in porous media, Adv. Water Resources, 30 (2007), pp. 1696-1710.

[39] C.J. Van Duijn and M.J. De Neef, Similarity solution for capillary redistribution of two phases in a porous medium with a single discontinuity, Adv. Water Resources, 21 (1998), pp. 451-461.

[40] T. Warburton and J.S. Hesthaven, On the constants in hp-finite element trace inverse inequalities, Comput. Methods Appl. Mech. Engrg., 192 (2003), pp. 2765-2773.

[41] M.F. WheELER, An elliptic collocation-finite element method with interior penalties, SIAM J. Numer. Anal., 15 (1978), pp. 152-161.

[42] J. YANG AND Y. Chen, A priori error analysis of a discontinuous Galerkin approximation for a kind of compressible miscible displacement problems, Sci. China Math., 53 (2010), pp. 2679-2696.

Copyright (C) by SIAM. Unauthorized reproduction of this article is prohibited. 